Supporting information:

\title{
A Sulfitylation-Oxidation Protocol for the Preparation of Sulfates
}

M. Huibers, Álvaro Manuzi, Floris P. J. T. Rutjes and Floris L. van Delft*

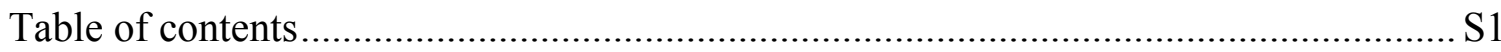

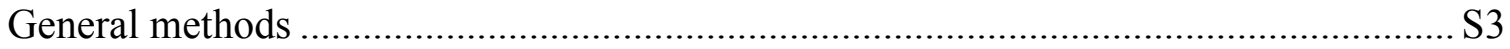

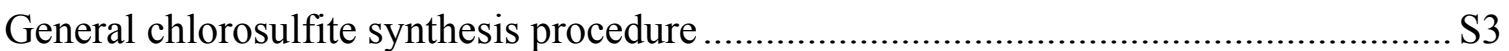

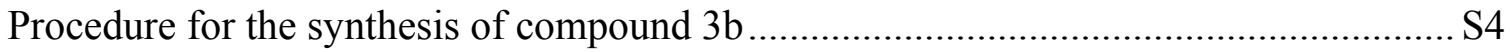

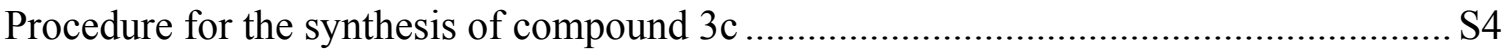

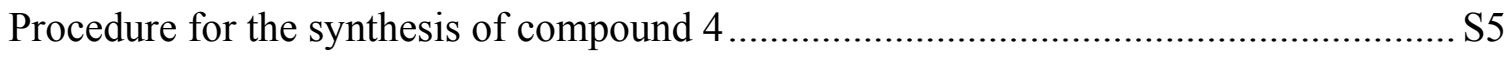

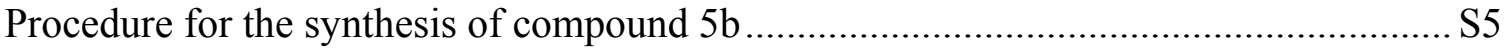

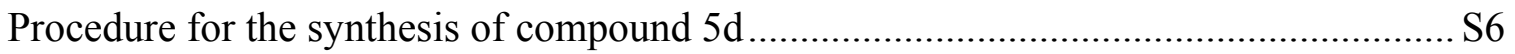

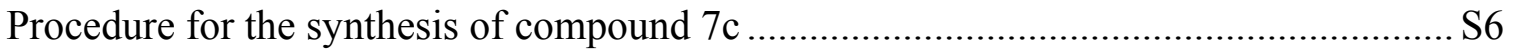

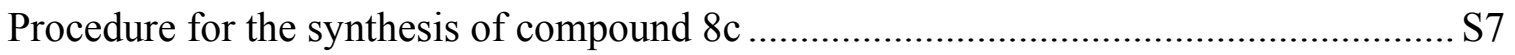

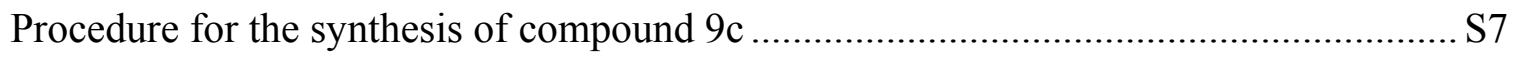

Procedure for the synthesis of compound 10c ............................................................ S8

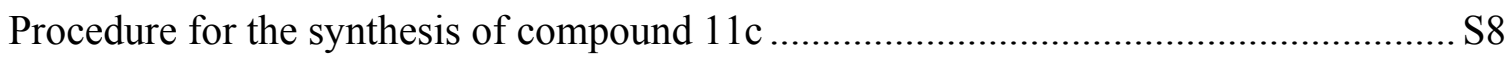

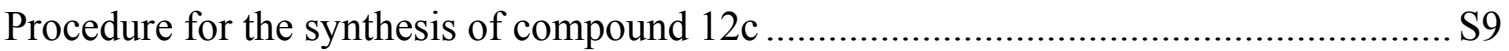

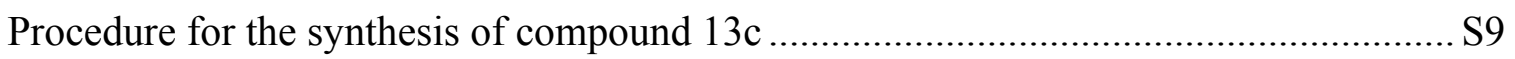

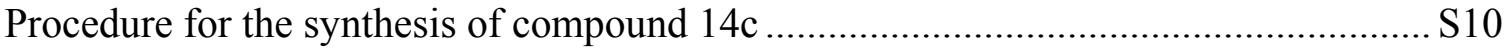

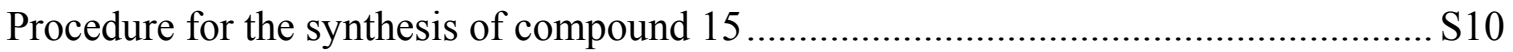

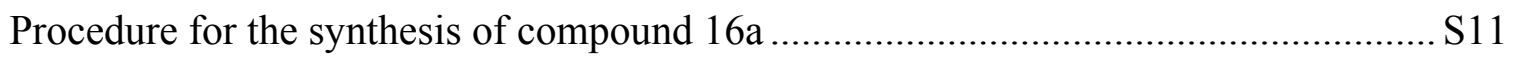

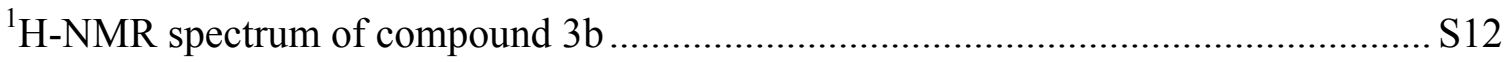




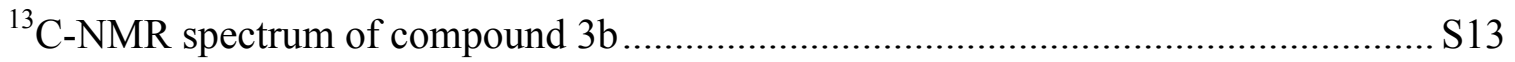

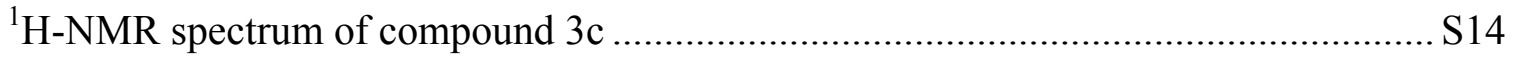

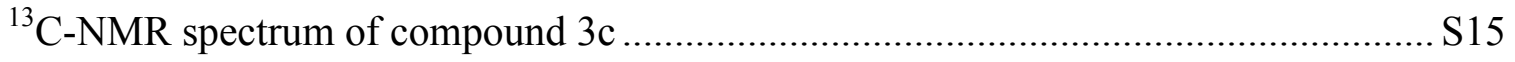

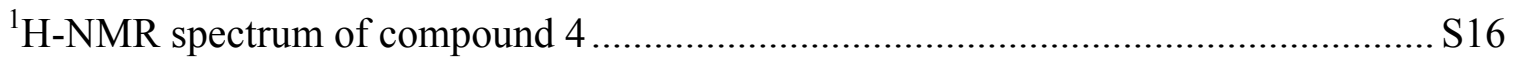

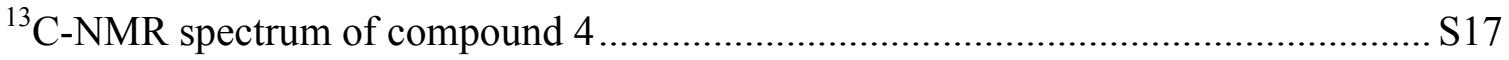

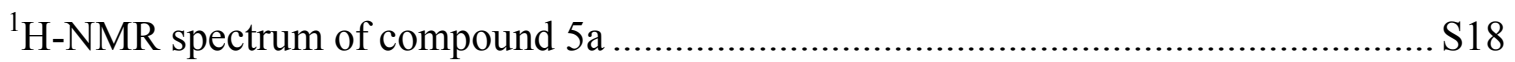

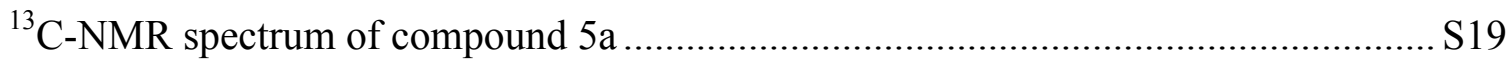

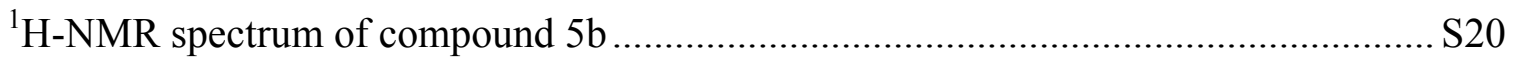

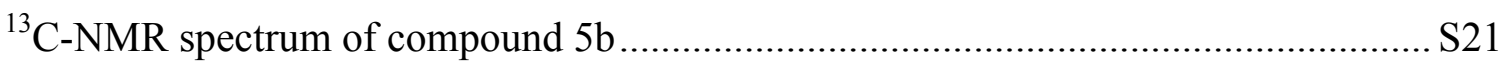

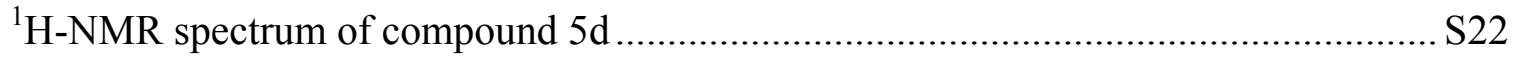

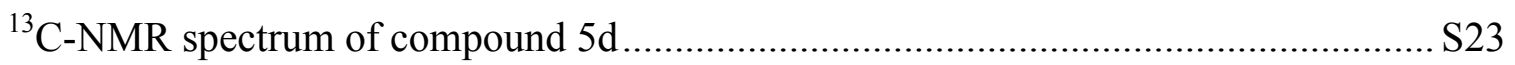

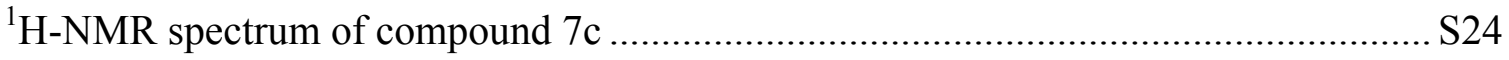

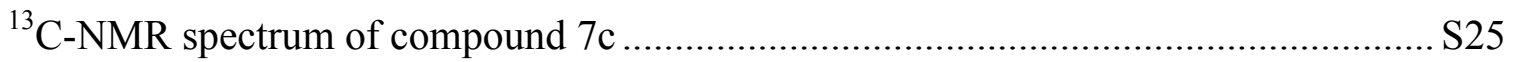

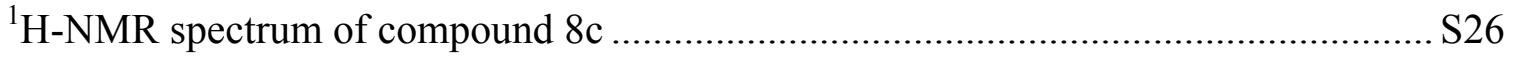

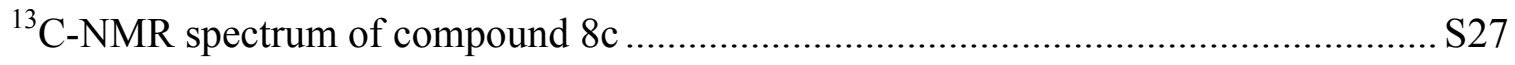

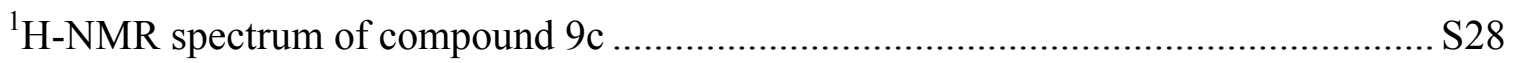

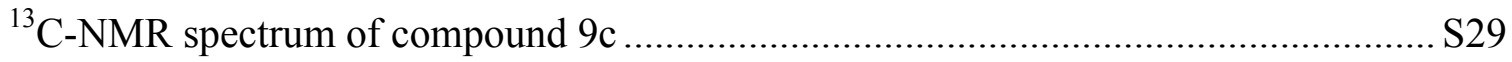

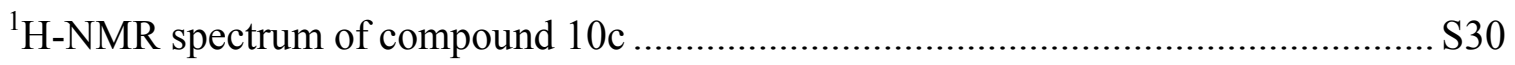

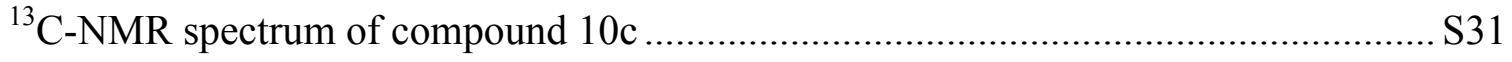

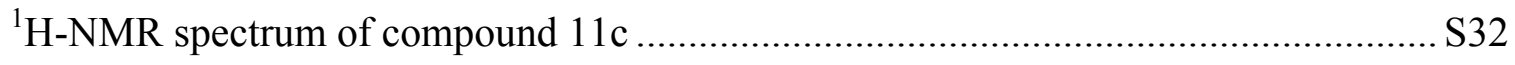

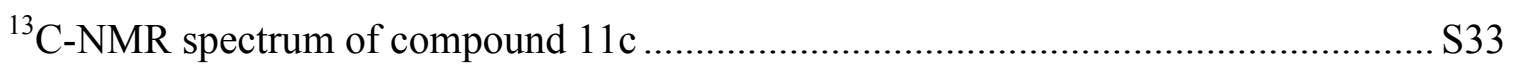

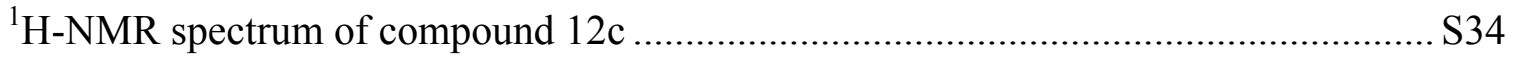

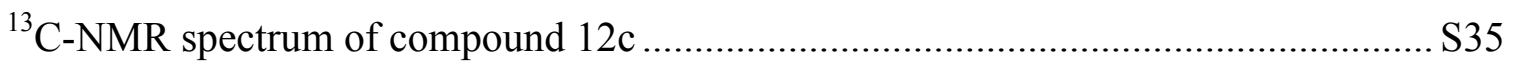




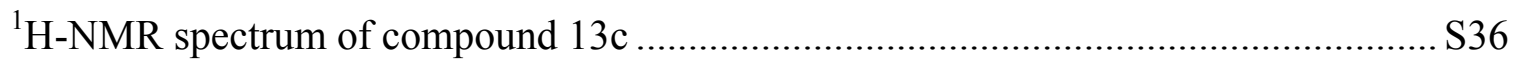

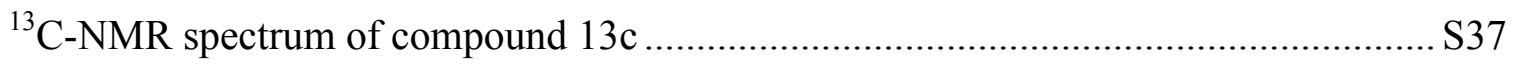

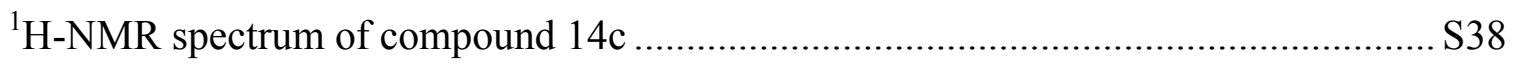

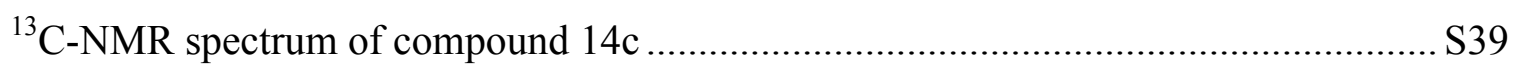

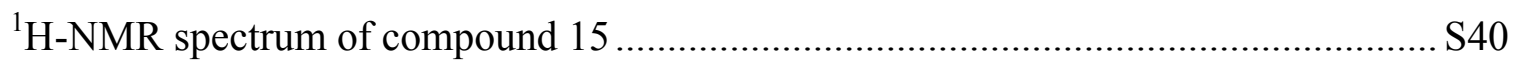

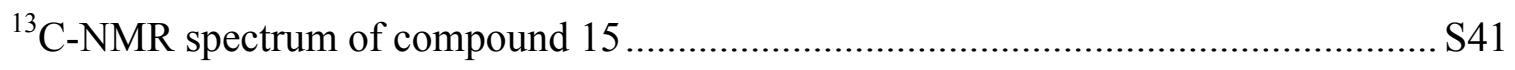

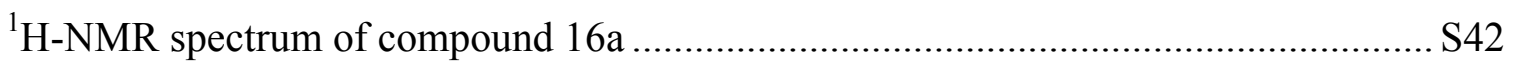

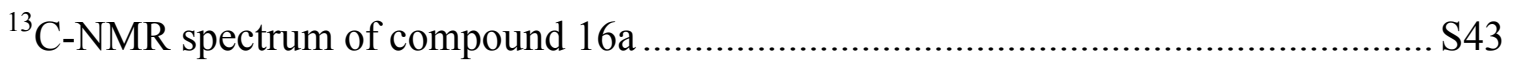

\section{General methods}

Standard syringe techniques were applied for the transfer of dry solvents and air- or moisture sensitive reagents. Compounds on TLC plates were visualized with ammonium molybdate solution, potassium permanganate, or UV light. Flash chromatography was carried out using silica gel $(0.035-0.070 \mathrm{~mm}$, ca $6 \mathrm{~nm}$ pore diameter $)$. Solvents were distilled from appropriate drying agents prior to use. Unless stated otherwise, all chemicals were purchased and used as such.

\section{General chlorosulfite synthesis procedure}

Thionyl chloride is put in a three-necked flask (neat) connected to two sequential traps with $10 \mathrm{M}$ aqueous $\mathrm{NaOH}$. A little nitrogen pressure is applied so the gas bubbles out of the last trap at moderate speed, to make sure the hydrochloric acid gas byproduct leaves the reaction mixture quickly enough. At $0^{\circ} \mathrm{C}$ the alcohol is slowly added dropwise over 
the course of several hours. After addition the solution is stirred one or two days at room temperature, after which a vacuum distillation is performed.

\section{$(R, S)-2,3-O$-isopropylidene-glycerol 1- ethyl sulfate (3b)}

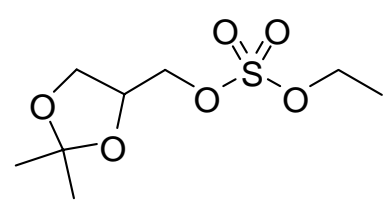

Sulfite diester $\mathbf{2 b}$ (159 $\mathrm{mg}, 0.71 \mathrm{mmol}$ ), sodium periodate (309 $\mathrm{mg}, 1.44 \mathrm{mmol}$ ) and ruthenium trichloride (cat.) were reacted as described in the general sulfite oxidation procedure,

yielding $\mathbf{3 b}$ as a colorless oil (162 mg, $95 \%$ ). IR $v_{\max }$ film: $\left(\mathrm{cm}^{-1}\right)$ 1376, 1199, 922. ${ }^{1} \mathrm{H}$ NMR ( $\left.\mathrm{CDCl}_{3}, 400 \mathrm{MHz}, \mathrm{ppm}\right): \delta 4.38(\mathrm{q}, 2 \mathrm{H}, J=7.1 \mathrm{~Hz}), 4.36(\mathrm{~m}, 1 \mathrm{H}), 4.22(\mathrm{~d}, 2 \mathrm{H}, J=$ $5.4 \mathrm{~Hz}), 4.11(\mathrm{dd}, 1 \mathrm{H}, J=6.5,8.8 \mathrm{~Hz}), 3.83(\mathrm{dd}, 1 \mathrm{H}, J=5.4,8.8 \mathrm{~Hz}), 1.44(\mathrm{q}, 3 \mathrm{H}, J=$ $0.7 \mathrm{~Hz}), 1.44$ (t, $3 \mathrm{H}, J=7.1 \mathrm{~Hz}), 1.37(\mathrm{q}, 3 \mathrm{H}, J=0.7 \mathrm{~Hz}) .{ }^{13} \mathrm{C} \mathrm{NMR}\left(\mathrm{CDCl}_{3}, 300 \mathrm{MHz}\right.$, ppm) $\delta: 110.2,72.7,71.9,70.0,65.8,26.4,25.1,14.5$. HRMS (EI) $\mathrm{m} / z$ calcd for $\mathrm{C}_{8} \mathrm{H}_{17} \mathrm{O}_{6} \mathrm{~S}(\mathrm{M}+\mathrm{H})^{+}:$241.0746, found: 241.0748 .

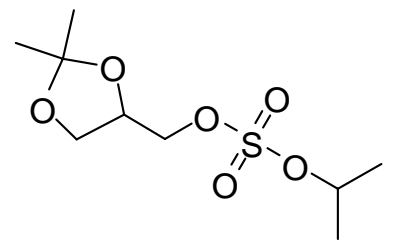

\section{$(R, S)-2,3-O$-isopropylidene-glycerol 1- isopropyl sulfate (3c)}

Sulfite diester $2 \mathbf{c}$ (407 $\mathrm{mg}, 1.71 \mathrm{mmol})$, sodium periodate (735

$\mathrm{mg}, 3.44 \mathrm{mmol}$ ) and ruthenium trichloride (cat.) were reacted as described in the general sulfite oxidation procedure, yielding $\mathbf{3 c}$ as a colorless oil (381 mg, 88\%). IR $v_{\max }$ film: $\left(\mathrm{cm}^{-1}\right) 1381,1195,918 .{ }^{1} \mathrm{H}$ NMR $\left(\mathrm{CDCl}_{3}, 400 \mathrm{MHz}, \mathrm{ppm}\right): \delta$ $4.94(\mathrm{qq}, 1 \mathrm{H}, J=6.3,6.3 \mathrm{~Hz}), 4.38(\mathrm{dq}, 1 \mathrm{H}, J=5.4,6.4 \mathrm{~Hz}), 4.19(\mathrm{dd}, 2 \mathrm{H}, J=0.7$, $5.4 \mathrm{~Hz}), 4.10(\mathrm{dd}, 1 \mathrm{H}, J=6.5 .8 .8 \mathrm{~Hz}), 3.84(\mathrm{dd}, 1 \mathrm{H}, J=5.4,8.8 \mathrm{~Hz}), 1.45(\mathrm{~d}, 6 \mathrm{H}, J=$ 6.3Hz), 1.43 (bq, $3 \mathrm{H}, J=0.6 \mathrm{~Hz}), 1.36(\mathrm{bq}, 3 \mathrm{H}, J=0.6 \mathrm{~Hz}) \cdot{ }^{13} \mathrm{C} \mathrm{NMR}\left(\mathrm{CDCl}_{3}, 300 \mathrm{MHz}\right.$, 
ppm) $\delta: 110.2,81.1,72.8,71.6,65.9,26.6,25.1,22.4$. HRMS (EI) $\mathrm{m} / z$ calcd for $\mathrm{C}_{9} \mathrm{H}_{19} \mathrm{O}_{6} \mathrm{~S}(\mathrm{M}+\mathrm{H})^{+}: 255,0902$, found: 255,0900 .

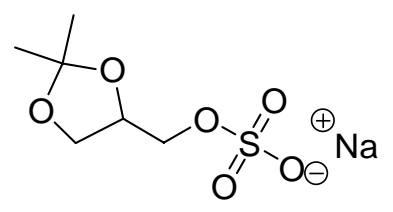

\section{$(R, S)-2,3-O$-isopropylidene-glycerol 1-sulfate, sodium salt (4)}

Sulfate diester $\mathbf{3 b}$ (402 $\mathrm{mg}, 1.67 \mathrm{mmol})$ and sodium iodide (252 $\mathrm{mg}, 1.68 \mathrm{mmol}$ ) were reacted as described in the general sulfate deprotection procedure, yielding 4 as a white solid (390 mg, 100\%). IR $v_{\max }$ film: $\left(\mathrm{cm}^{-1}\right)$ 1225, 1074, 996. ${ }^{1} \mathrm{H}$ NMR ( $\left.\mathrm{D}_{2} \mathrm{O}, 400 \mathrm{MHz}, \mathrm{ppm}\right): \delta 4.52$ (dtd, $\left.1 \mathrm{H}, J=3.8,5.9,6.6 \mathrm{~Hz}\right)$, $4.21(\mathrm{dd}, 1 \mathrm{H}, J=6.8,8.9 \mathrm{~Hz}), 4.16(\mathrm{dd}, 1 \mathrm{H}, J=3.8,10.9 \mathrm{~Hz}), 4.07(\mathrm{dd}, 1 \mathrm{H}, J=5.9$ $10.9 \mathrm{~Hz}) 3.92(\mathrm{dd}, 1 \mathrm{H}, J=5.9,8.9 \mathrm{~Hz}), 1.49$ (s, 3H), $1.42(\mathrm{~s}, 3 \mathrm{H}) .{ }^{13} \mathrm{C} \mathrm{NMR}\left(\mathrm{H}_{2} \mathrm{O}, 300\right.$ MHz, ppm): $\delta 109.9,73.2,67.8,65.0,25.0,23.7$. HRMS (ESI) $m / z$ calcd for $\mathrm{C}_{6} \mathrm{H}_{11} \mathrm{O}_{6} \mathrm{~S}$ (M-Na): 211.0276, found: 211.0279.<smiles>CC(C)OS(=O)Cl</smiles>

\section{Isopropyl chlorosulfite (5b)}

Thionyl chloride $(120 \mathrm{~g}, 1.01 \mathrm{~mol})$ and isopropyl alcohol $(50.0 \mathrm{~g}, 0.832$ mol) were reacted as described in the general chlorosulfite synthesis procedure. Distillation was performed at $50{ }^{\circ} \mathrm{C}(40 \mathrm{mbar})$, yielding $\mathbf{5 b}$ as a colorless liquid $(\sim 78 \%)$. ${ }^{1} \mathrm{H}$ NMR $\left(\mathrm{CDCl}_{3}, 400 \mathrm{MHz}, \mathrm{ppm}\right): \delta 5.50(\mathrm{qq}, 1 \mathrm{H}, J=6.3,6.3 \mathrm{~Hz}), 1.52(\mathrm{~d}, 3 \mathrm{H}, J=$ 6.3Hz), 1.47 (d, $3 \mathrm{H}, J=6.3 \mathrm{~Hz}) .{ }^{13} \mathrm{C} \mathrm{NMR}\left(\mathrm{CDCl}_{3}, 300 \mathrm{MHz}, \mathrm{ppm}\right)$ 8: 76.8, 68.3, 24.0, 23.8, 23.2. Note: peaks of the rapidly formed degradation product isopropyl chloride are also present in the ${ }^{13} \mathrm{C}$ spectrum. 
Neopentyl chlorosulfite (5d)

$\mathrm{Cl}^{-} \mathrm{S}_{-} \mathrm{O}$ Thionyl chloride $(43.5 \mathrm{~g}, 366 \mathrm{mmol})$ and neopentyl alcohol $(26.9 \mathrm{~g}, 305$ mmol) were reacted as described in the general chlorosulfite synthesis procedure with exception of a vacuum distillation. Instead excess thionyl chloride was distilled off selectively at $40{ }^{\circ} \mathrm{C}$ (50 mbar) yielding $\mathbf{5 d}$ as a colorless liquid ( 95\%). ${ }^{1} \mathrm{H} \mathrm{NMR}\left(\mathrm{CDCl}_{3}\right.$, $400 \mathrm{MHz}, \mathrm{ppm}): \delta 4.25(\mathrm{~d}, 1 \mathrm{H}, J=9.4 \mathrm{~Hz}), 3.97(\mathrm{~d}, 1 \mathrm{H}, J=9.4 \mathrm{~Hz}), 1.02(\mathrm{~s}, 9 \mathrm{H}) .{ }^{13} \mathrm{C}$ $\operatorname{NMR}\left(\mathrm{CDCl}_{3}, 300 \mathrm{MHz}, \mathrm{ppm}\right):$ 76.0, 31.6, 26.4 .

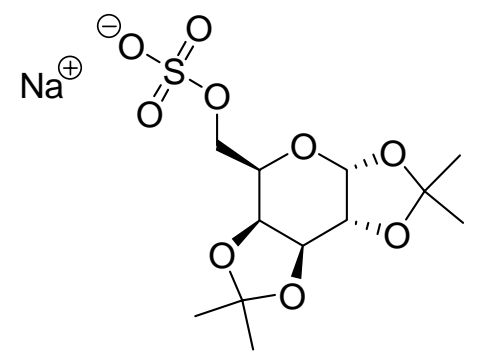

1,2:3,4-Di-O-isopropylidene- $\alpha$-D-galactopyranose 6-
sulfate, sodium salt (7c)
Sulfate diester $7 \mathbf{b}(1255 \mathrm{mg}, 3.41 \mathrm{mmol})$ and sodium iodide
$(512 \mathrm{mg}, 3.42 \mathrm{mmol})$ were reacted as described in the general sulfate deprotection procedure, yielding 7c as a white solid (1234 $\mathrm{mg}, 100 \%)$. $[\alpha]_{\mathrm{D}}{ }^{20}-40$ (c 1.02; MeOH). IR $v_{\max }$ film: $\left(\mathrm{cm}^{-1}\right) 1649,1389,1255,1217,1074,999 .{ }^{1} \mathrm{H}$ NMR (D $2 \mathrm{O}, 400 \mathrm{MHz}, \mathrm{ppm}): \delta 5.68(\mathrm{~d}, 1 \mathrm{H}, J=4.9 \mathrm{~Hz}), 4.83(\mathrm{dd}, 1 \mathrm{H}, J=2.3,7.9 \mathrm{~Hz})$, $4.58(\mathrm{dd}, 1 \mathrm{H}, J=2.5,5.0 \mathrm{~Hz}), 4.50(\operatorname{app~d}, 1 \mathrm{H}, 7.9 \mathrm{~Hz}), 4.30-4.21(\mathrm{~m}, 2 \mathrm{H}), 4.12(\mathrm{dd}, 1 \mathrm{H}, J$ $=7.7,10.8 \mathrm{~Hz}), 1.60(\mathrm{~s}, 3 \mathrm{H}), 1.50(\mathrm{~s}, 3 \mathrm{H}), 1.42(\mathrm{~s}, 6 \mathrm{H}) .{ }^{13} \mathrm{C} \mathrm{NMR}\left(\mathrm{D}_{2} \mathrm{O}, 300 \mathrm{MHz}, \mathrm{ppm}\right)$

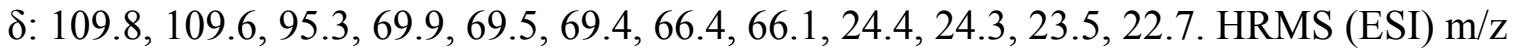
calcd for $\mathrm{C}_{12} \mathrm{H}_{19} \mathrm{O}_{9} \mathrm{~S}(\mathrm{M}-\mathrm{Na})^{-}: 339.0750$, found: 339.0781 . 


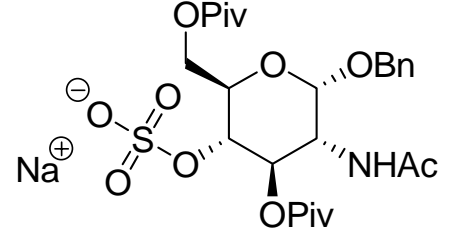

\section{1-O-Benzyl-2-deoxy-2-acetamido-3,6-di-O-pivaloyl $\alpha$-D-} glucopyranose 4-sulfate, sodium salt (8c)

Sulfate diester $\mathbf{8 b}(28.0 \mathrm{mg}, 0.048 \mathrm{mmol})$ and sodium iodide procedure, yielding $8 \mathrm{c}$ as a white solid $(27.3 \mathrm{mg}, 99 \%) .[\alpha]_{\mathrm{D}}{ }^{20}+48$ (c $\left.0.18 ; \mathrm{MeOH}\right)$. IR $v_{\max }$ film: $\left(\mathrm{cm}^{-1}\right) 1717,1644,1488,1449,1280,1155,1074,991,610 .{ }^{1} \mathrm{H}$ NMR $\left(\mathrm{CD}_{3} \mathrm{OD}\right.$, $400 \mathrm{MHz}, \mathrm{ppm}): \delta 7.42-7.28(\mathrm{~m}, 5 \mathrm{H}), 5.30(\mathrm{dd}, 1 \mathrm{H}, J=9.1,11.0 \mathrm{~Hz}), 4.79(\mathrm{~d}, 1 \mathrm{H}, J=$ $3.6 \mathrm{~Hz}), 3.78(\mathrm{~d}, 1 \mathrm{H}, J=12.0 \mathrm{~Hz}), 4.54(\mathrm{~d}, 1 \mathrm{H}, J=11.9 \mathrm{~Hz}), 4.53(\mathrm{dd}, 1 \mathrm{H}, J=2.1$, $12.0 \mathrm{~Hz}), 4.44(\mathrm{dd}, 1 \mathrm{H}, J=2.1,12.0 \mathrm{~Hz}), 4.32-4.23(\mathrm{~m}, 2 \mathrm{H}), 4.05(\mathrm{ddd}, 1 \mathrm{H}, J=2.1,6.5$, 10.1Hz), $3.68(\mathrm{~s}, 1 \mathrm{H}), 1.87(\mathrm{~s}, 3 \mathrm{H}), 1.22(\mathrm{~s}, 9 \mathrm{H}), 1.17(\mathrm{~s}, 9 \mathrm{H}) .{ }^{13} \mathrm{C}$ NMR $\left(\mathrm{CD}_{3} \mathrm{OD}, 300\right.$ MHz, ppm) $\delta: 129.6,129.5,129.2,97.2,75.7,72.2,70.5,70.4,64.8,53.1,27.7,27.7$, 22.5. HRMS (ESI) $m / z$ calcd for $\mathrm{C}_{25} \mathrm{H}_{36} \mathrm{NO}_{11} \mathrm{~S}(\mathrm{M}-\mathrm{Na})^{-}:$558.2009, found: 558.2050.

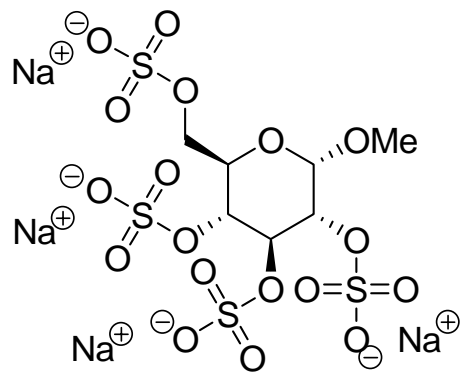

\section{1-O-Methyl- $\alpha$-D-glucopyranose 2,3,4,6-tetrasulfate,} tetrasodium salt $(9 \mathrm{c})$

Tetrasulfate diester $9 \mathbf{b}(66.6 \mathrm{mg}, 0.11 \mathrm{mmol})$ and sodium iodide $(88.0 \mathrm{mg}, 0.58 \mathrm{mmol})$ were reacted as described in the general sulfate deprotection procedure, yielding $9 \mathrm{c}$ as a white solid (63.4 mg, 99\%). $[\alpha]_{\mathrm{D}}^{20}+133\left(\mathrm{c} 0.12 ; \mathrm{H}_{2} \mathrm{O}\right)$. IR $v_{\max }$ film: $\left(\mathrm{cm}^{-1}\right) 1644,1233$, 1074, 987. ${ }^{1} \mathrm{H}$ NMR ( $\left.\mathrm{D}_{2} \mathrm{O}, 400 \mathrm{MHz}, \mathrm{ppm}\right): \delta 5.21(\mathrm{~d}, 1 \mathrm{H}, J=3.6 \mathrm{~Hz}), 4.72-4.61(\mathrm{~m}$, 2H), $4.46(\mathrm{ddd}, 1 \mathrm{H}, J=1.1,3.7,9.8 \mathrm{~Hz}), 4.37-4.32(\mathrm{~m}, 1 \mathrm{H}), 4.23-4.14(\mathrm{~m}, 2 \mathrm{H}), 3.52(\mathrm{~d}$, 3H, 1.1Hz). 13C NMR (D2O, $300 \mathrm{MHz}, \mathrm{ppm}) \delta:$ 96.3, 75.3, 74.5, 73.8, 68.0, 66.9, 54.9. HRMS (ESI) $m / z$ calcd for $\mathrm{C}_{7} \mathrm{H}_{10} \mathrm{Na}_{3} \mathrm{O}_{18} \mathrm{~S}_{4}(\mathrm{M}-\mathrm{Na})^{-}:$578.8443, found: 578.8494 . 


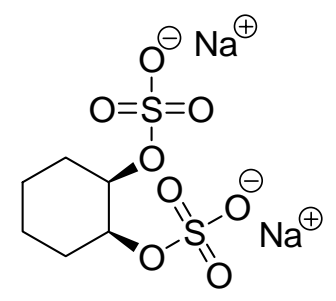

(R,S)-Cyclohexane-1,2-diol 1,2-disulfate, disodium salt (10c)

Disulfate diester 10b (883 mg, $2.66 \mathrm{mmol})$ and sodium iodide (798 $\mathrm{mg}, 5.33 \mathrm{mmol}$ ) were reacted as described in the general sulfate deprotection procedure, yielding $10 \mathrm{c}$ as a white solid $(847 \mathrm{mg}$, 99\%). IR $v_{\max }$ film: $\left(\mathrm{cm}^{-1}\right) 1644,1221,1069,1001 .{ }^{1} \mathrm{H}$ NMR $\left(\mathrm{D}_{2} \mathrm{O}, 400 \mathrm{MHz}, \mathrm{ppm}\right): \delta$ 4.62-4.57 (m, 2H), 2.07-1.97 (m, 2H), 1.79-1.62 (m, 4H), 1.49-1.39 (m, 2H). ${ }^{13} \mathrm{C}$ NMR $\left(\mathrm{D}_{2} \mathrm{O}, 300 \mathrm{MHz}, \mathrm{ppm}\right) \delta$ : 77.4, 27.2, 20.4. HRMS (ESI) $\mathrm{m} / z$ calcd for $\mathrm{C}_{6} \mathrm{H}_{10} \mathrm{NaO}_{8} \mathrm{~S}_{2}(\mathrm{M}-$ Na): 296,972 , found: 296,974 .

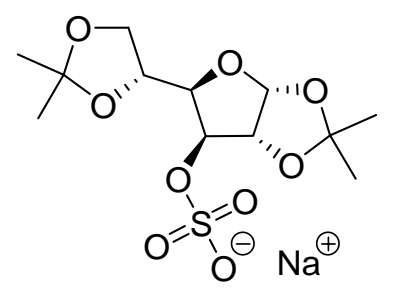

\section{1,2:5,6-Di-O-isopropylidene-a-D-glucofuranose 3-sulfate,} sodium salt (11c)

Sulfate diester 11b (101 mg, $0.27 \mathrm{mmol})$ and sodium iodide (42 $\mathrm{mg}, 0.28 \mathrm{mmol}$ ) were reacted as described in the general sulfate deprotection procedure, yielding 11c as a white solid (98 mg, 99\%). $[\alpha]_{\mathrm{D}}^{20}-10$ (c 0.45 ; MeOH). IR $v_{\max }$ film: $\left(\mathrm{cm}^{-1}\right) 1649,1376,1243,1225,1014,858 .{ }^{1} \mathrm{H}$ NMR $\left(\mathrm{D}_{2} \mathrm{O}, 400\right.$ MHz, ppm): $\delta 6.12(\mathrm{~d}, 1 \mathrm{H}, J=3.7 \mathrm{~Hz}), 5.01(\mathrm{~d}, 1 \mathrm{H}, J=3.7 \mathrm{~Hz}), 4.87(\mathrm{~d}, 1 \mathrm{H}, J=2.5 \mathrm{~Hz})$, 4.58-4.53 (m, 2H) , 4.21-4.15 (m, 1H), 4.11-4.05 (m, 1H), $1.56(\mathrm{~s}, 3 \mathrm{H}), 1.49(\mathrm{~s}, 3 \mathrm{H}), 1.41$ (s, 3H), 1.40 (s, 3H). ${ }^{13} \mathrm{C}$ NMR ( $\left.\mathrm{D}_{2} \mathrm{O}, 300 \mathrm{MHz}, \mathrm{ppm}\right) \delta: 112.8,108.8,104.3,82.2,79.4$, 79.1, 72.4, 64.4, 25.2, 24.9, 24.8, 23.4. HRMS (ESI) $m / z$ calcd for $\mathrm{C}_{12} \mathrm{H}_{19} \mathrm{O}_{9} \mathrm{~S}(\mathrm{M}-\mathrm{Na})^{-}$: 339.0750, found: 339.0782 . 


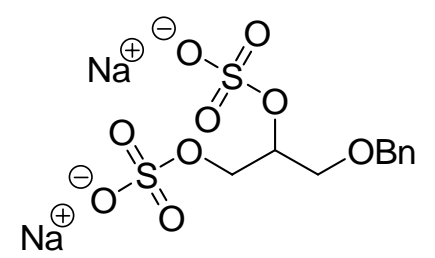

\section{3-Benzylglycerol 1,2-disulfate, disodium salt (12c)}

Disulfate diester 12b (225 mg, $0.56 \mathrm{mmol})$ and sodium iodide (220 $\mathrm{mg}, 1.47 \mathrm{mmol})$ were reacted as described in the general sulfate deprotection procedure, yielding $\mathbf{1 2 c}$ as a white solid $(217 \mathrm{mg}, 100 \%)$. IR $v_{\max }$ film: $\left(\mathrm{cm}^{-1}\right) 1632,1226,1052,1026,936 .{ }^{1} \mathrm{H}$ NMR $\left(\mathrm{D}_{2} \mathrm{O}, 400 \mathrm{MHz}, \mathrm{ppm}\right): \delta$ 7.50-7.39 (m, 5H), 4.75 (dtt, $1 \mathrm{H}, J=1.8,4.8,4.8 \mathrm{~Hz}), 4.68$ (ddd, $2 \mathrm{H}, J=1.4,11.6,18.8 \mathrm{~Hz}), 4.29$ (dddd, $2 \mathrm{H}, J=1.9,4.8,10.9,20.2 \mathrm{~Hz}), 3.86(\mathrm{dddd}, 2 \mathrm{H}, J=1.6,5.0,11.6,29.1 \mathrm{~Hz}) \cdot{ }^{13} \mathrm{C}$ NMR ( $\left.\mathrm{D}_{2} \mathrm{O}, 300 \mathrm{MHz}, \mathrm{ppm}\right) \delta$ : 136.6, 128.1, 127.9, 127.7, 75.1, 72.9, 67.6, 66.2. HRMS (ESI) $m / z$ calcd for $\mathrm{C}_{10} \mathrm{H}_{12} \mathrm{O}_{9} \mathrm{~S}_{2}(\mathrm{M}-\mathrm{Na})^{-}: 362.9820$, found: 362.9837 .

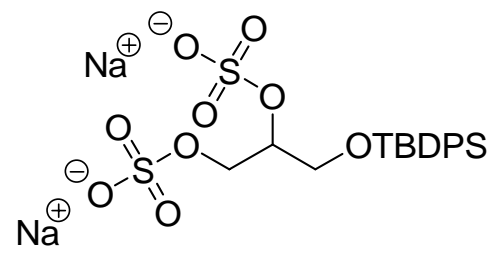

\section{3-(tert-Butyl diphenylsilyl)-glycerol 1,2-disulfate, disodium salt (13c)}

Disulfate diester 13b (796 mg, $1.46 \mathrm{mmol}$ ) and sodium iodide (460 $\mathrm{mg}, 3.07 \mathrm{mmol}$ ) were reacted as described in the general sulfate deprotection procedure, yielding $13 \mathbf{c}$ as a white solid (783mg, 100\%). IR $v_{\max }$ film: $\left(\mathrm{cm}^{-1}\right)$ 1640, 1372, 1216, 1203, 914. ${ }^{1} \mathrm{H}$ NMR $\left(\mathrm{D}_{2} \mathrm{O}, 400 \mathrm{MHz}, \mathrm{ppm}\right): \delta$ 7.84-7.79 (m, 4H), 7.59-7.48 (m, $6 \mathrm{H}), 4.60(\mathrm{tt}, 1 \mathrm{H}, J=4.9,4.9 \mathrm{~Hz}), 4.32(\mathrm{ddd}, 2 \mathrm{H}, J=4.7,10.6,22.7 \mathrm{~Hz}), 3.99$ (ddd, $2 \mathrm{H}, J$ $=5.0,11.3,17.9 \mathrm{~Hz}), 1.09(\mathrm{~s}, 9 \mathrm{H}) \cdot{ }^{13} \mathrm{C} \mathrm{NMR}\left(\mathrm{D}_{2} \mathrm{O}, 300 \mathrm{MHz}, \mathrm{ppm}\right) \delta: 135.2,132.6$, $129.8,127.6,127.4,76.3,65.7,61.5,25.6$, 17.9. HRMS (ESI) $\mathrm{m} / \mathrm{z}$ calcd for $\mathrm{C}_{19} \mathrm{H}_{24} \mathrm{NaO}_{9} \mathrm{~S}_{2} \mathrm{Si}(\mathrm{M}-\mathrm{Na})^{-}: 511.0529$, found: 511.0552 . 


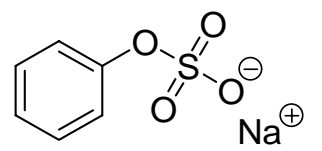

\section{Phenyl sulfate, sodium salt (14c)}

methanol, to which sodium methoxide $(33 \mathrm{mg}, 0.61 \mathrm{mmol}$ ) was added. After stirring for 1 hour the solvent was evaporated. The residue was flash chromatographed $\left(\mathrm{MeCN} / \mathrm{H}_{2} \mathrm{O}\right)$, yielding 14c as a white solid (56 mg, 100\%). IR $v_{\max }$ film: $\left(\mathrm{cm}^{-1}\right) 616,603 .{ }^{1} \mathrm{H}$ NMR $\left(\mathrm{D}_{2} \mathrm{O}, 300 \mathrm{MHz}, \mathrm{ppm}\right): \delta$ 7.55-7.45 (m, 2H), 7.41-7.33 (m, 3H). ${ }^{13} \mathrm{C}$ NMR $\left(\mathrm{D}_{2} \mathrm{O}, 300\right.$ MHz, ppm) $\delta: 150.6,129.4,125.8,121.0$. HRMS (ESI) $m / z$ calcd for $\mathrm{C}_{6} \mathrm{H}_{5} \mathrm{O}_{4} \mathrm{~S}(\mathrm{M}-\mathrm{Na})^{-}$: 172.9909, found: 172.9910 .

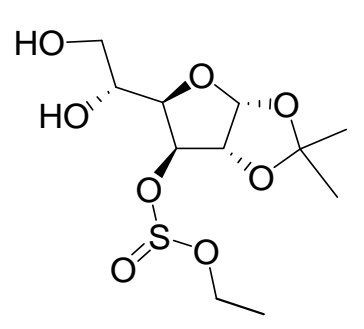

\section{1,2-O-isopropylidene- $\alpha$-D-glucofuranose 3-[(R,S)-ethyl sulfite]}

Sulfite 11a (570 mg, $1.62 \mathrm{mmol}$ ) was dissolved in $\mathrm{AcOH} / \mathrm{H}_{2} \mathrm{O}$

$1 / 1$, and heated to $50{ }^{\circ} \mathrm{C}$ for $3 \mathrm{~h}$. The solvents were evaporated, and the crude product was flash chromatographed (heptane / EtOAc 1/ 1) to yield $\mathbf{1 5}$ as a white solid (442 mg, $88 \%$; 2:3 mixture of diastereomers). IR $v_{\max }$ film: $\left(\mathrm{cm}^{-1}\right) 3469$, 1371, 1207, 1082, 1017. ${ }^{1} \mathrm{H}$ NMR $\left(\mathrm{CDCl}_{3}, 400 \mathrm{MHz}, \mathrm{ppm}\right): \delta 5.93(\mathrm{~d}, 1 \mathrm{H}, J=3.7 \mathrm{~Hz})$, $5.15+4.93(\operatorname{app~d} / \mathrm{td}, 1 \mathrm{H}, J=2.6 \mathrm{~Hz} / J=0.5,2.9 \mathrm{~Hz}), 4.64+4.58(\mathrm{~d}, 1 \mathrm{H}, J=3.8 \mathrm{~Hz})$, 4.34-4.09 (m, 2H), 3.94-3.68 (m, 3H), 3.33+3.07 (d / br s, $1 \mathrm{H}, J=4.7 \mathrm{~Hz}), 1.52+1.51(\mathrm{~s}$, $3 \mathrm{H}), 1.41+1.38(\mathrm{t}, 3 \mathrm{H}, J=7.1 \mathrm{~Hz}), 1.33+1.32(\operatorname{app~d}, 3 \mathrm{H}, J=0.5 \mathrm{~Hz}) .{ }^{13} \mathrm{C} \mathrm{NMR}\left(\mathrm{CDCl}_{3}\right.$, $300 \mathrm{MHz}, \mathrm{ppm}) \delta: 112.5+112.4,105.0+105.0,83.5,79.2+79.1,76.2+74.4,68.2+67.9$, 64.1+64.0, 60.8+59.5, 26.6, 26.2+26.2, 15.3+15.2. HRMS (ESI) $m / z$ calcd for $\mathrm{C}_{11} \mathrm{H}_{21} \mathrm{O}_{8} \mathrm{~S}$ $(\mathrm{M}+\mathrm{H})^{+}:$313.0957, found: 313.0950 . 


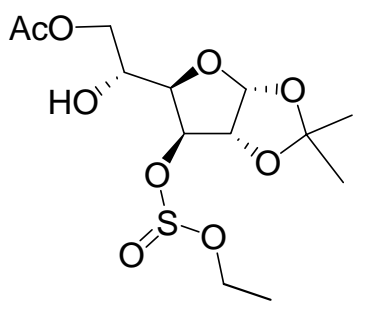

6-O-acetyl-1,2-O-isopropylidene- $\alpha$-D-glucofuranose 3-[(R,S)ethyl sulfite] (16a)

Sulfite 15 (20.8 mg, $0.076 \mathrm{mmol})$ and acetic anhydride $(30.4 \mathrm{mg}$, $0.30 \mathrm{mmol})$ were dissolved in dry pyridine. The solvent was evaporated after $1 \mathrm{~h}$, and ethyl acetate was added to the reaction mixture. Subsequent washing with water and brine, drying with $\mathrm{Na}_{2} \mathrm{SO}_{4}$, and filtration yielded the crude product. This was flash chromatographed (heptane / ethyl acetate 3 / 1) to yield 16a as a white solid (22.0 mg, 93\%; 4:5 mixture of diastereomers). IR $v_{\max }$ film: $\left(\mathrm{cm}^{-1}\right) 3490$, 1743, 1216, 1086, 1017. ${ }^{1} \mathrm{H}$ NMR $\left(\mathrm{CDCl}_{3}, 400 \mathrm{MHz}, \mathrm{ppm}\right): \delta 5.92+5.92(\mathrm{~d}, 1 \mathrm{H}, J=$ $3.7 \mathrm{~Hz}), 5.12+4.94(\mathrm{~d}, 1 \mathrm{H}, J=2.5 \mathrm{~Hz}), 4.63+4.59(\mathrm{~d}, 1 \mathrm{H}, J=3.7 \mathrm{~Hz}), 4.46+4.43(\mathrm{t}, 1 \mathrm{H}, J$ $=2.1 \mathrm{~Hz}), 4.32-4.09(\mathrm{~m}, 4 \mathrm{H}), 4.09-3.91(\mathrm{~m}, 1 \mathrm{H}), 3.24+2.98(\mathrm{~d}, 1 \mathrm{H}, J=4.6 \mathrm{~Hz})$, $2.11+2.10(\mathrm{~s}, 3 \mathrm{H}), 1.51+1.50(\mathrm{~s}, 3 \mathrm{H}), 1.40+1.37(\mathrm{t}, 3 \mathrm{H}, 7.1 \mathrm{~Hz}), 1.33+1.32(\mathrm{~s}, 3 \mathrm{H}) .{ }^{13} \mathrm{C}$ NMR $\left(\mathrm{CDCl}_{3}, 300 \mathrm{MHz}, \mathrm{ppm}\right) \delta: 112.6+112.6,105.2+105.1,83.6,78.9+78.8,75.9,74.2$, $66.9+66.5,66.4+66.2,60.9+59.7,26.7,26.4+26.4,20.9,15.4+15.3$. HRMS (ESI) $\mathrm{m} / \mathrm{z}$ calcd for $\mathrm{C}_{13} \mathrm{H}_{23} \mathrm{O}_{9} \mathrm{~S}(\mathrm{M}+\mathrm{H})^{+}: 355.1063$, found: 355.1071 . 


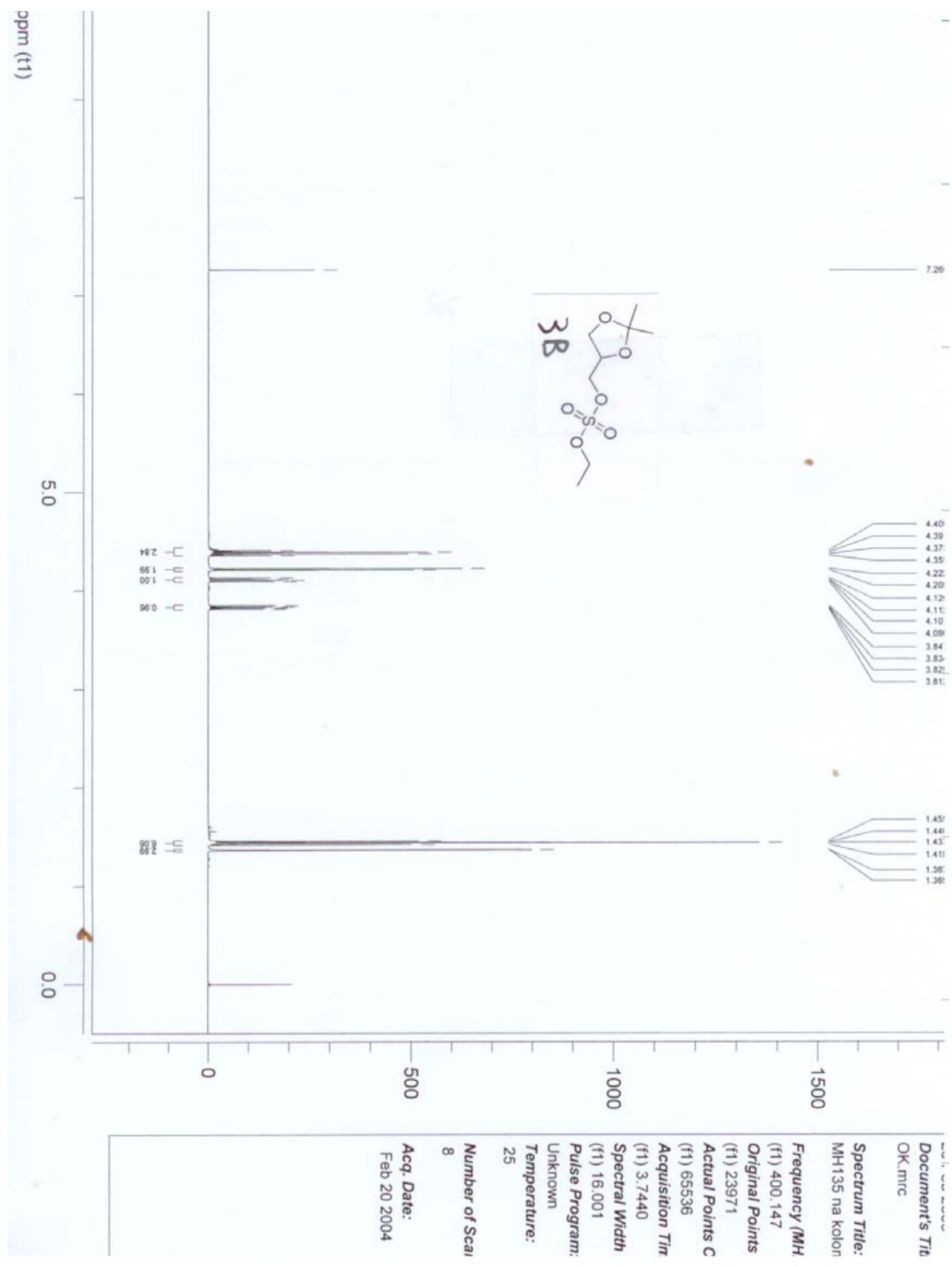




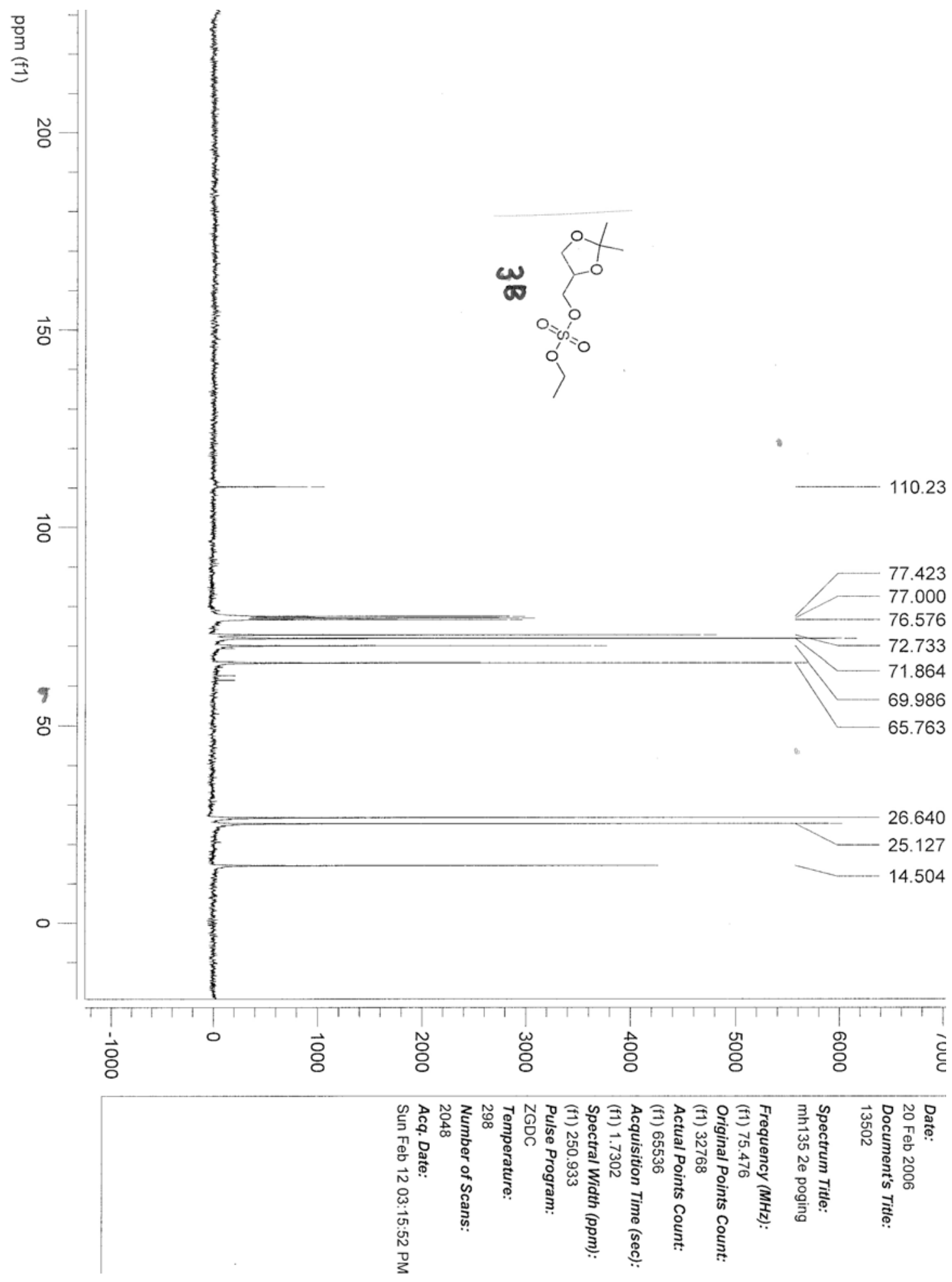




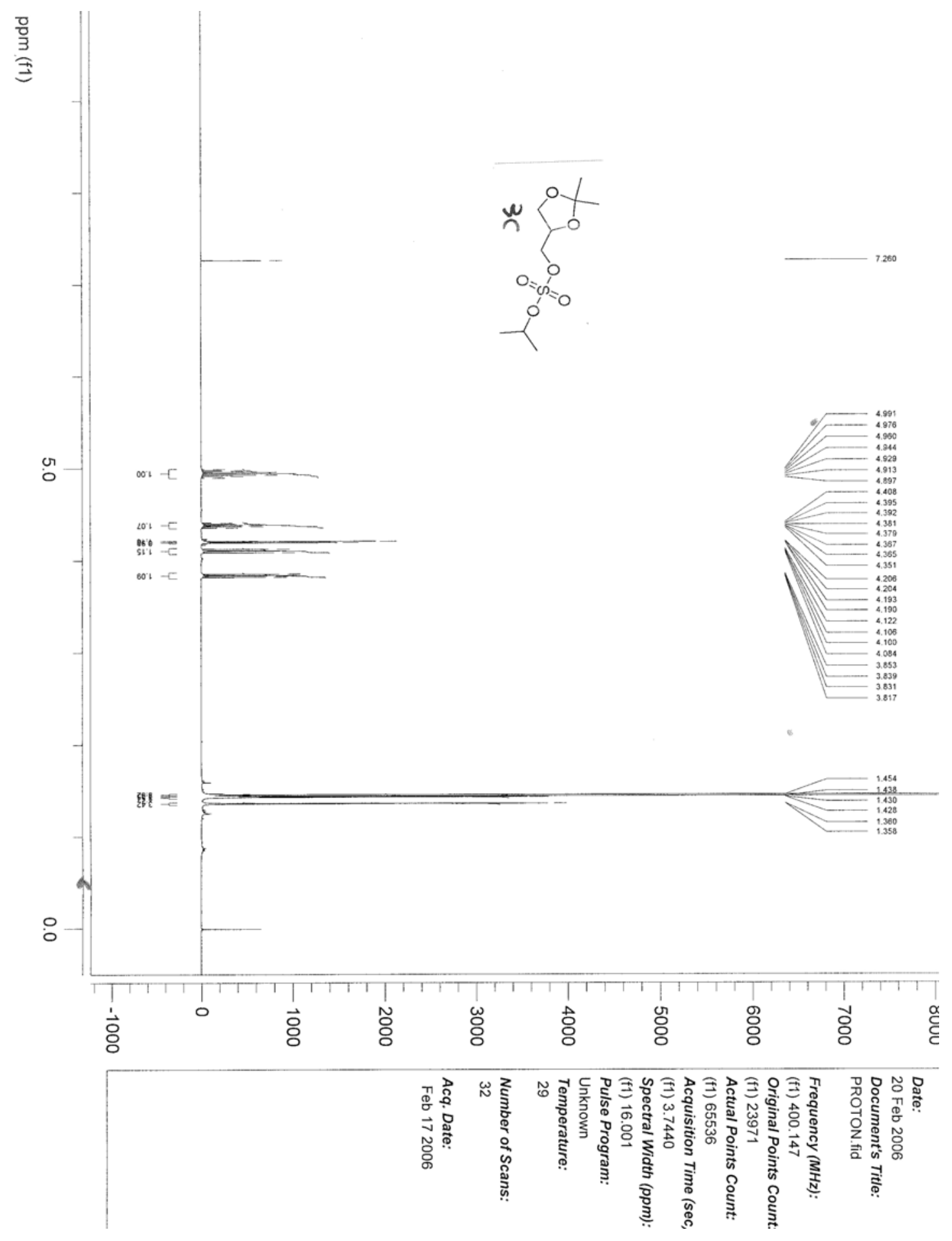




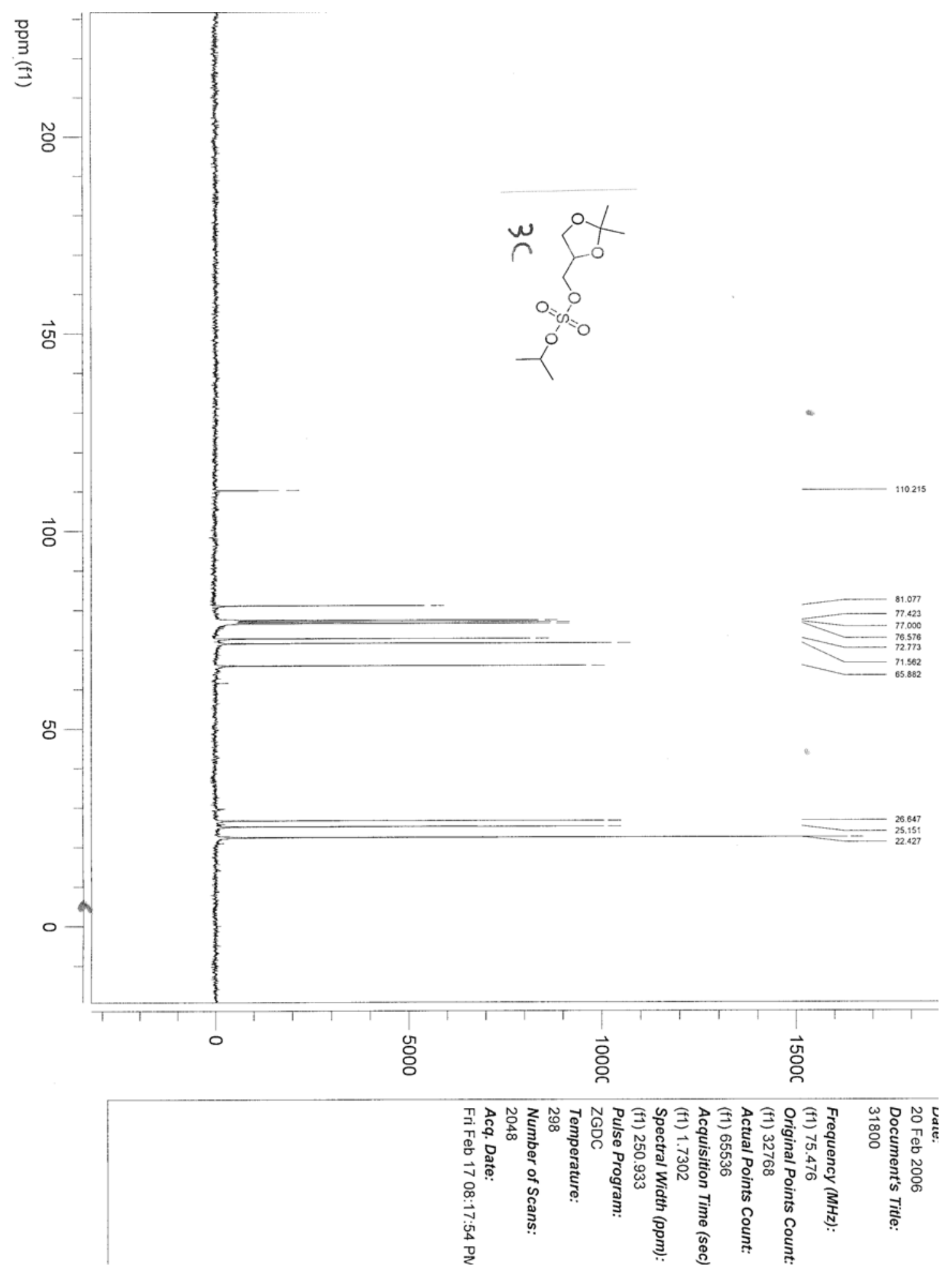




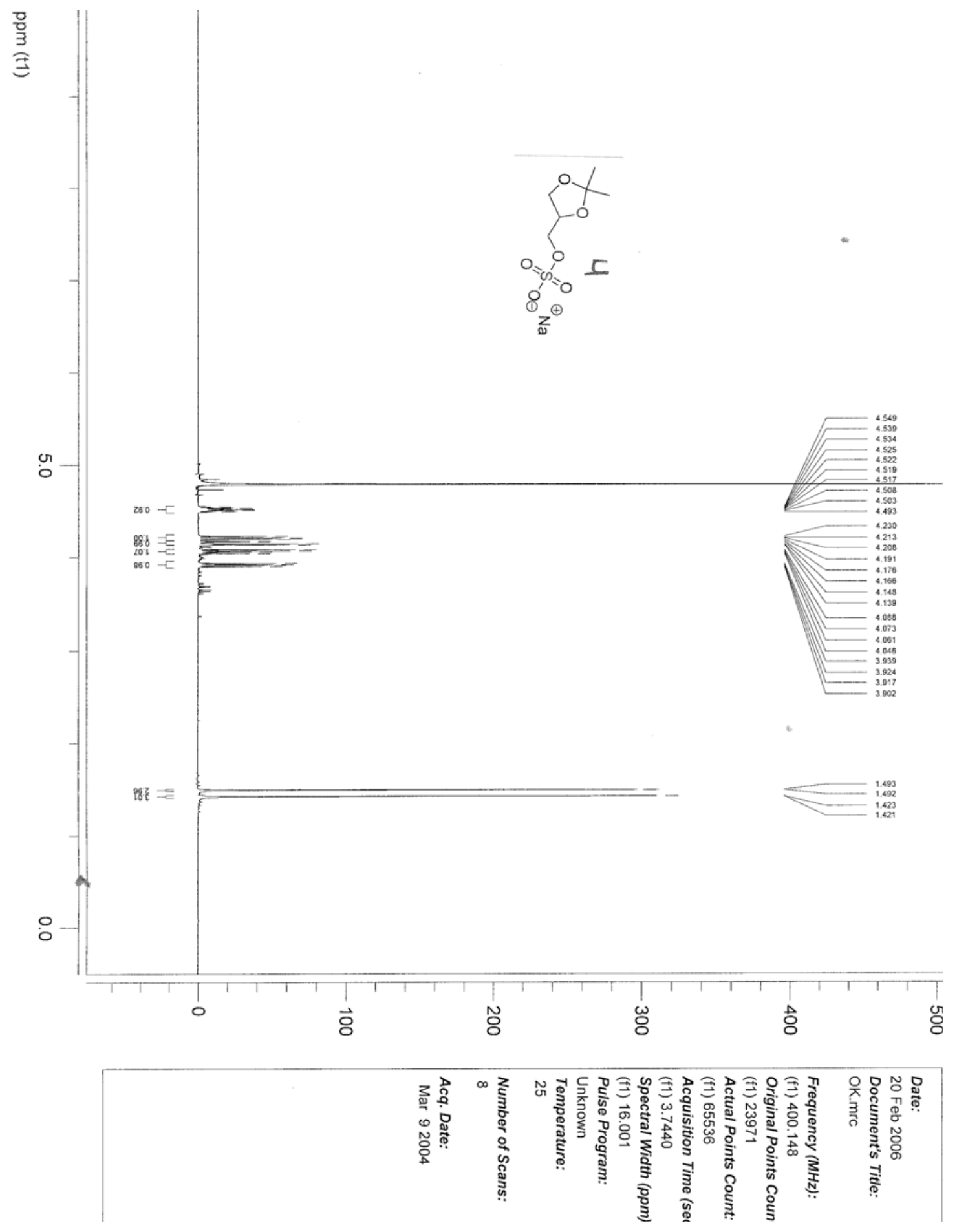




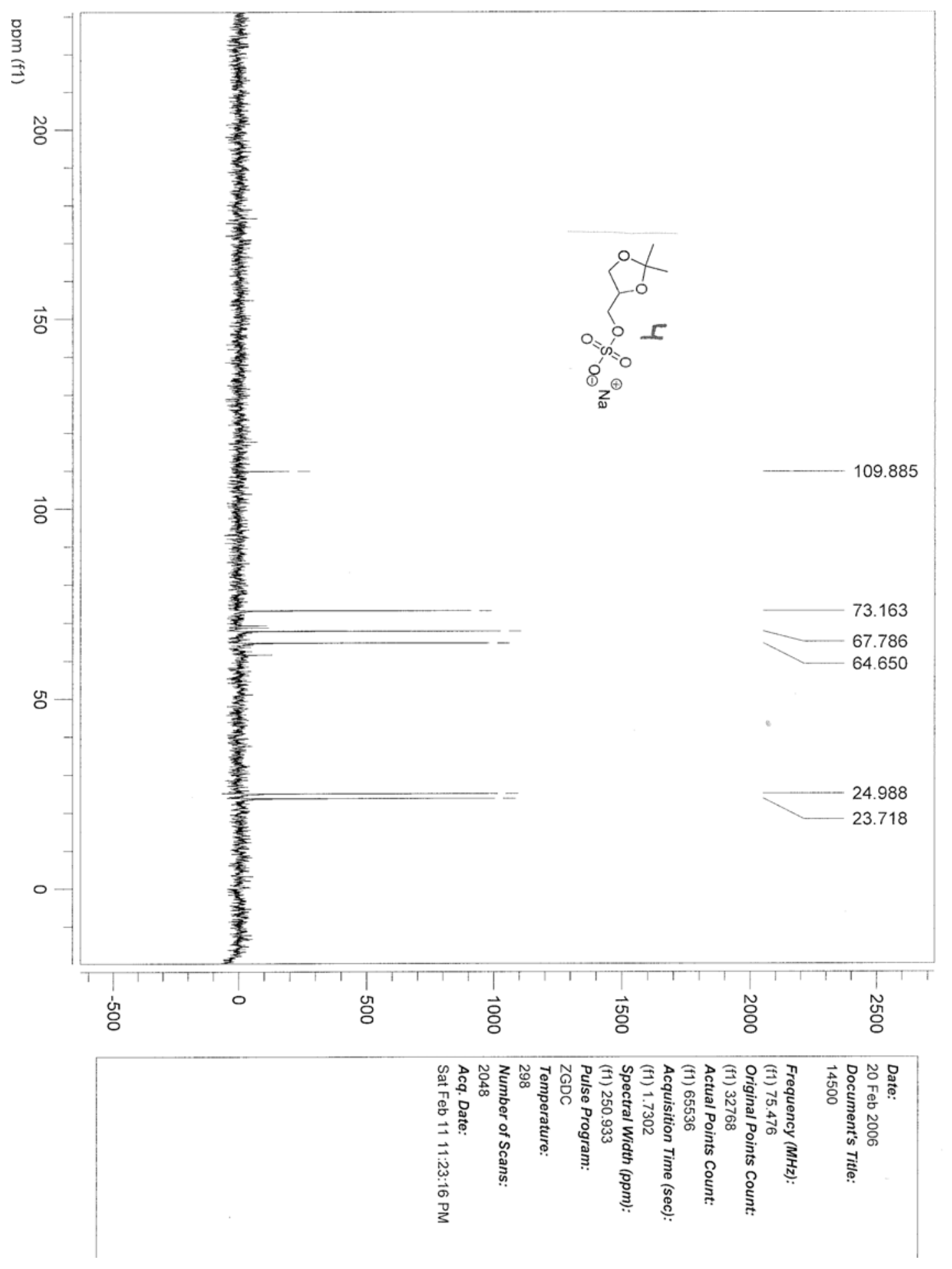



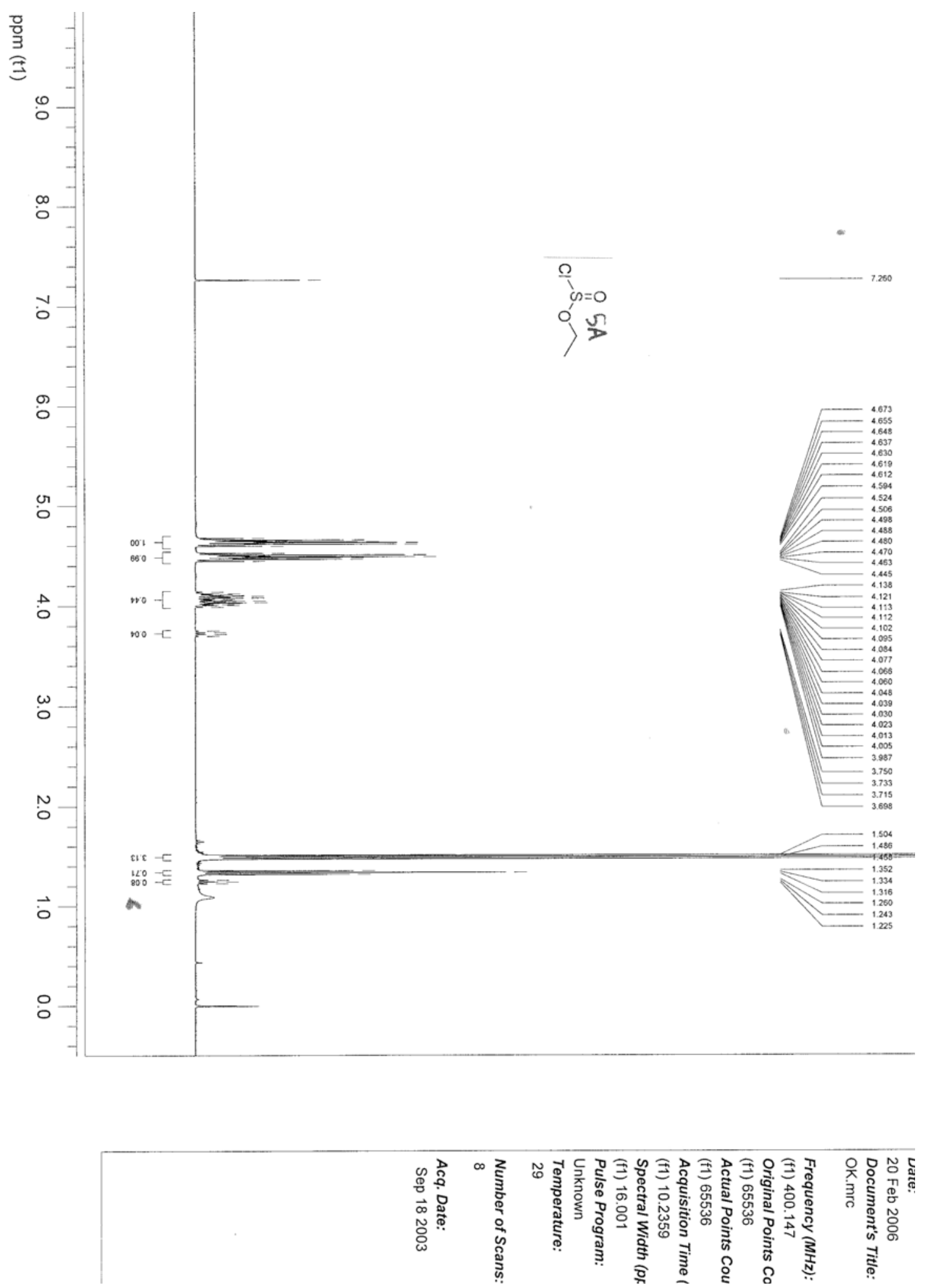

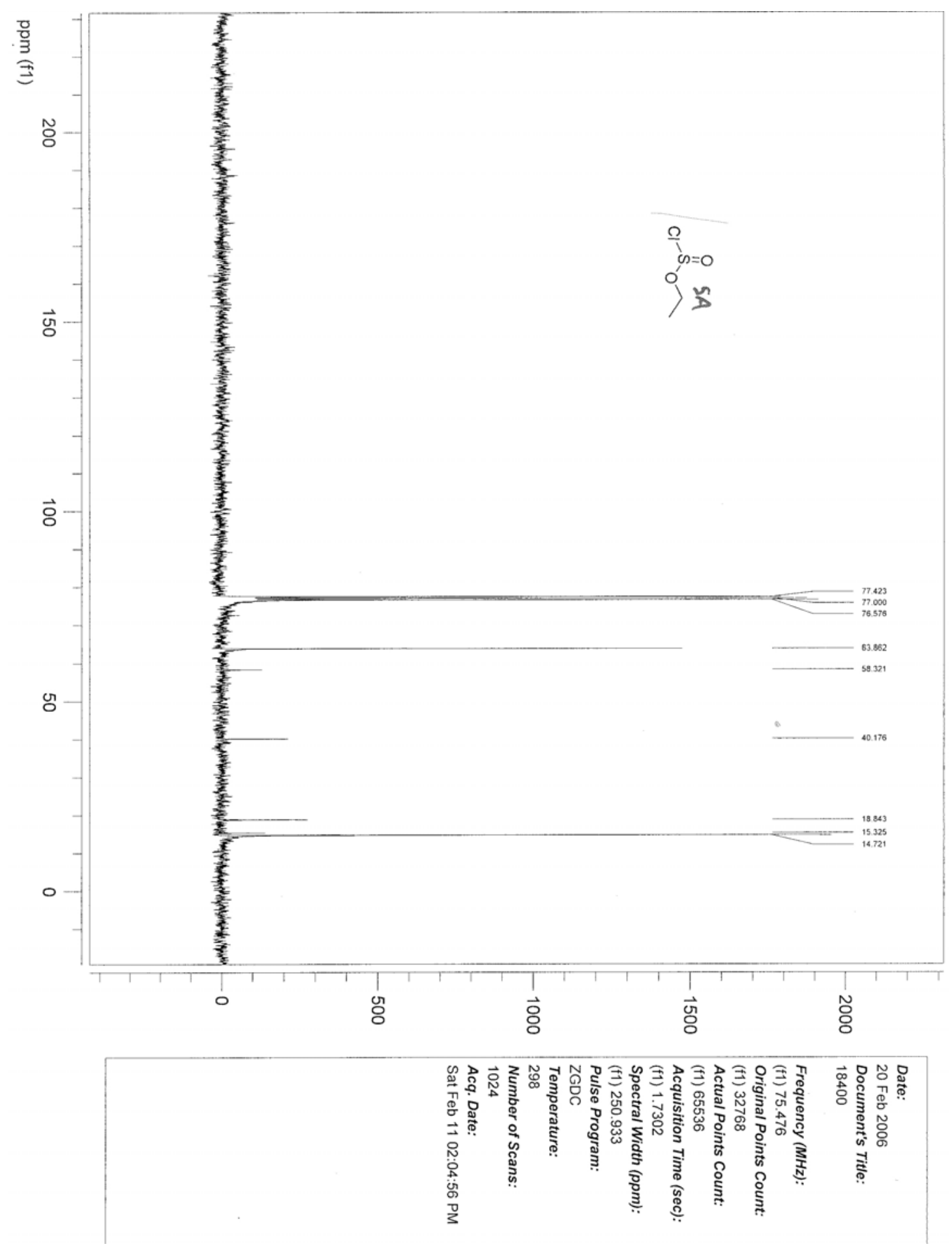


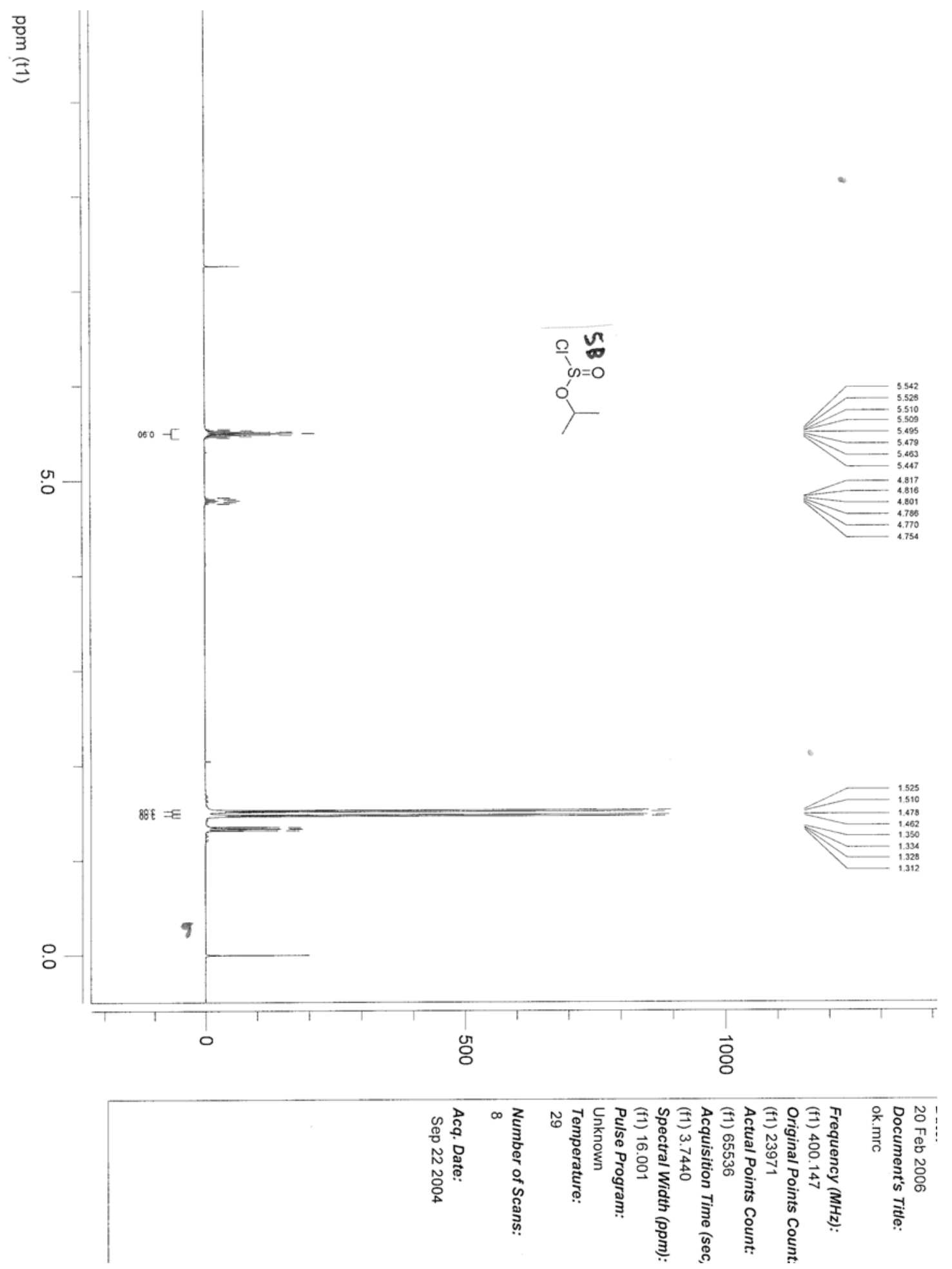




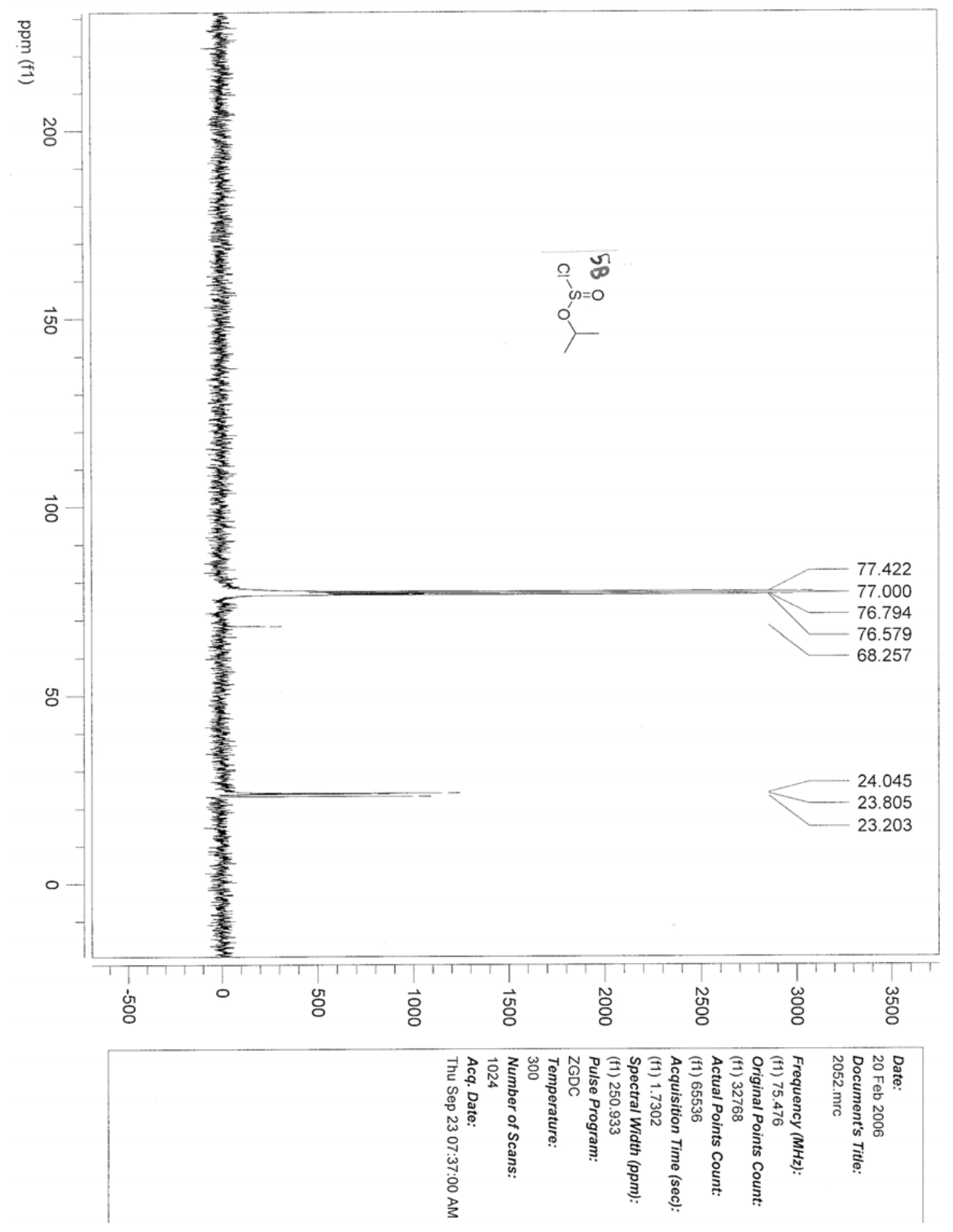




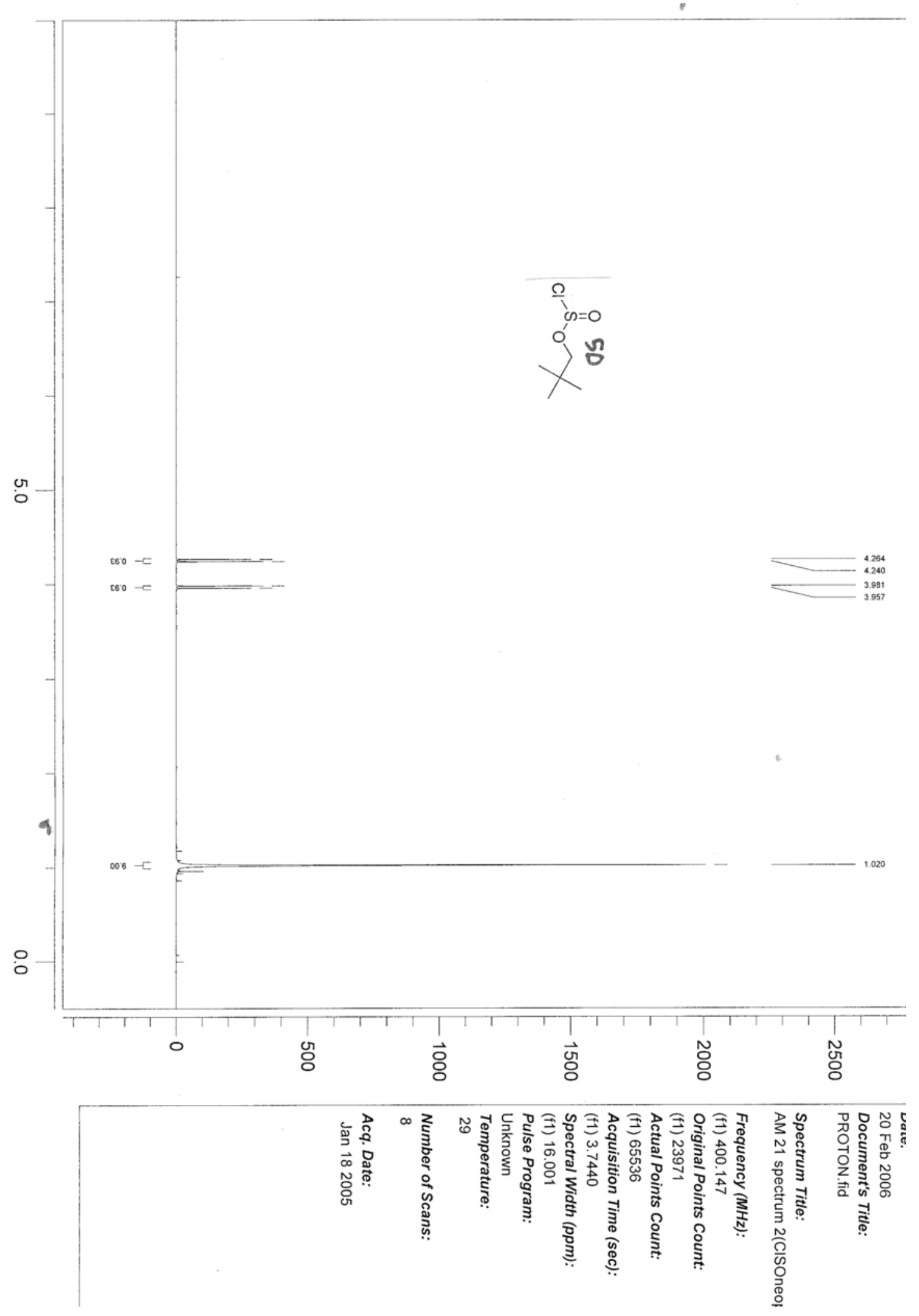




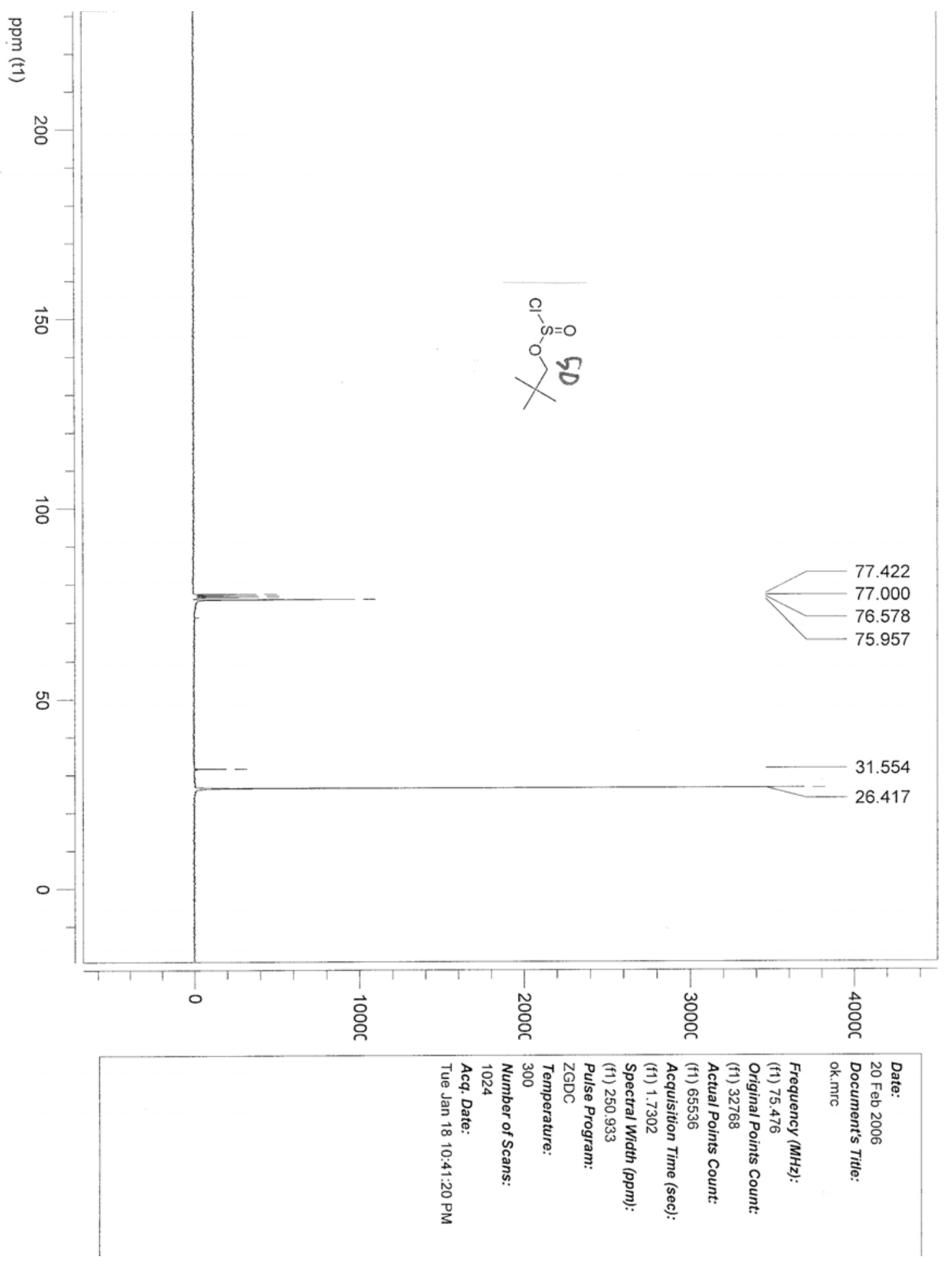




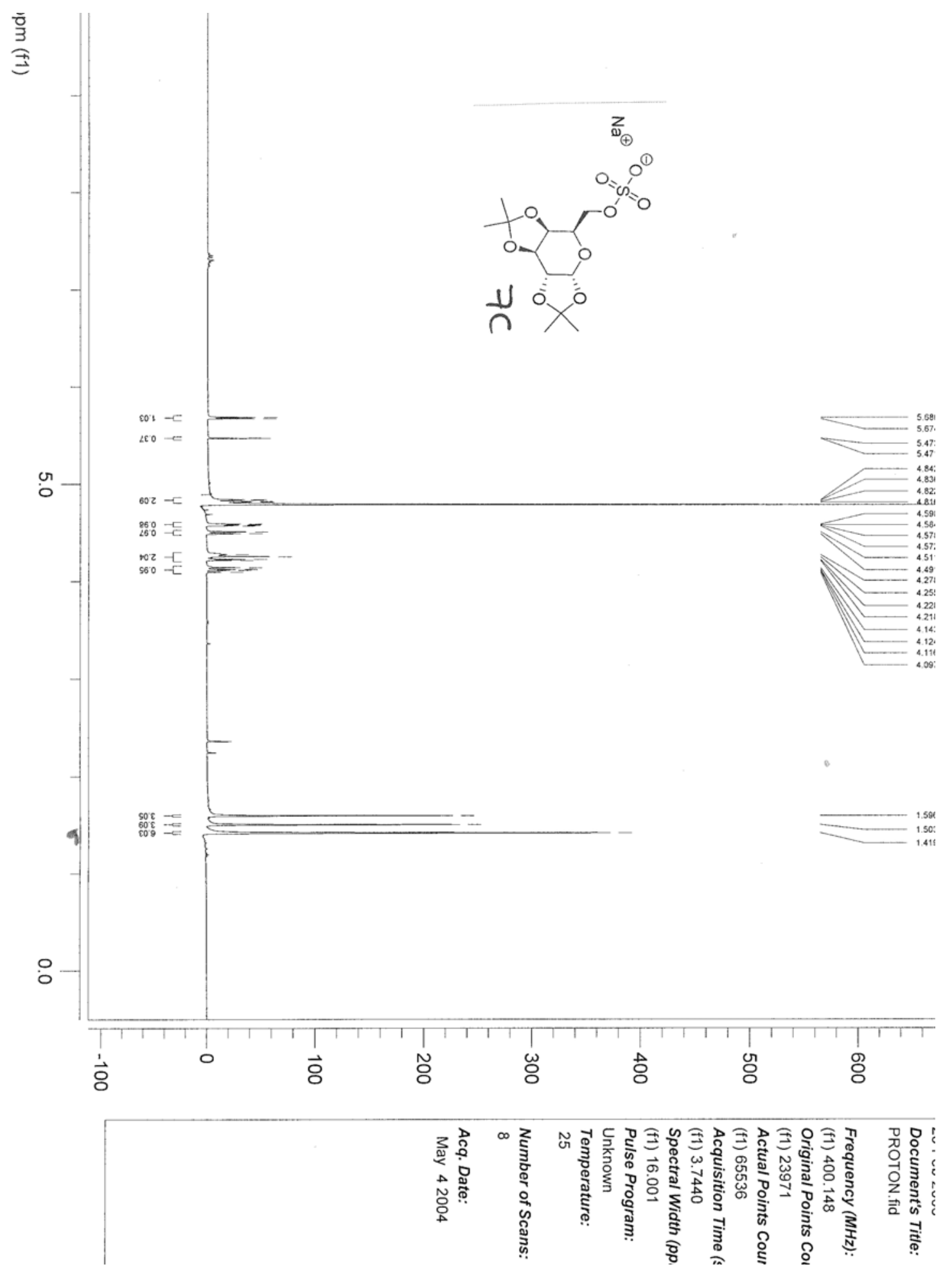




$$
\mathrm{E}
$$




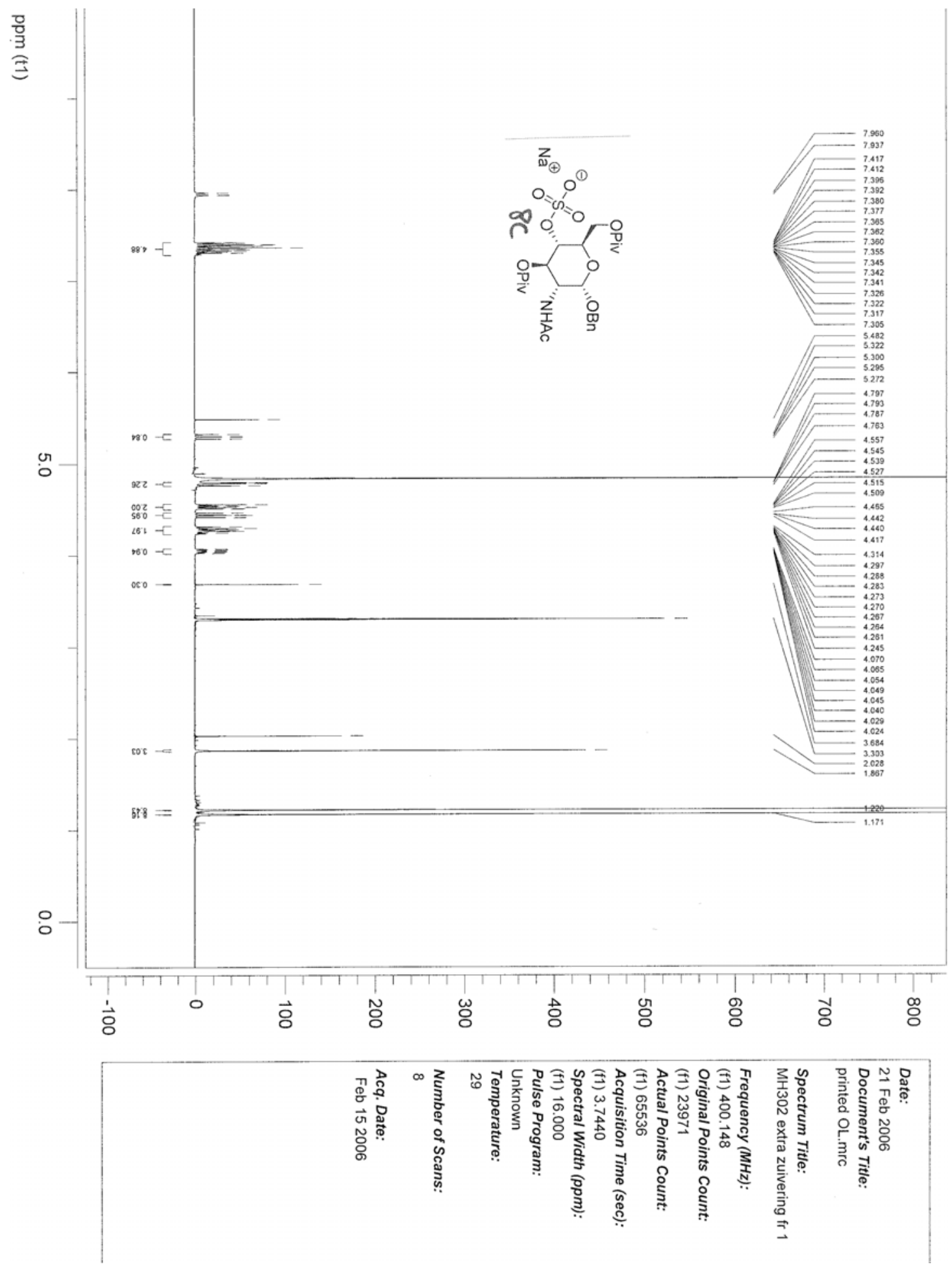



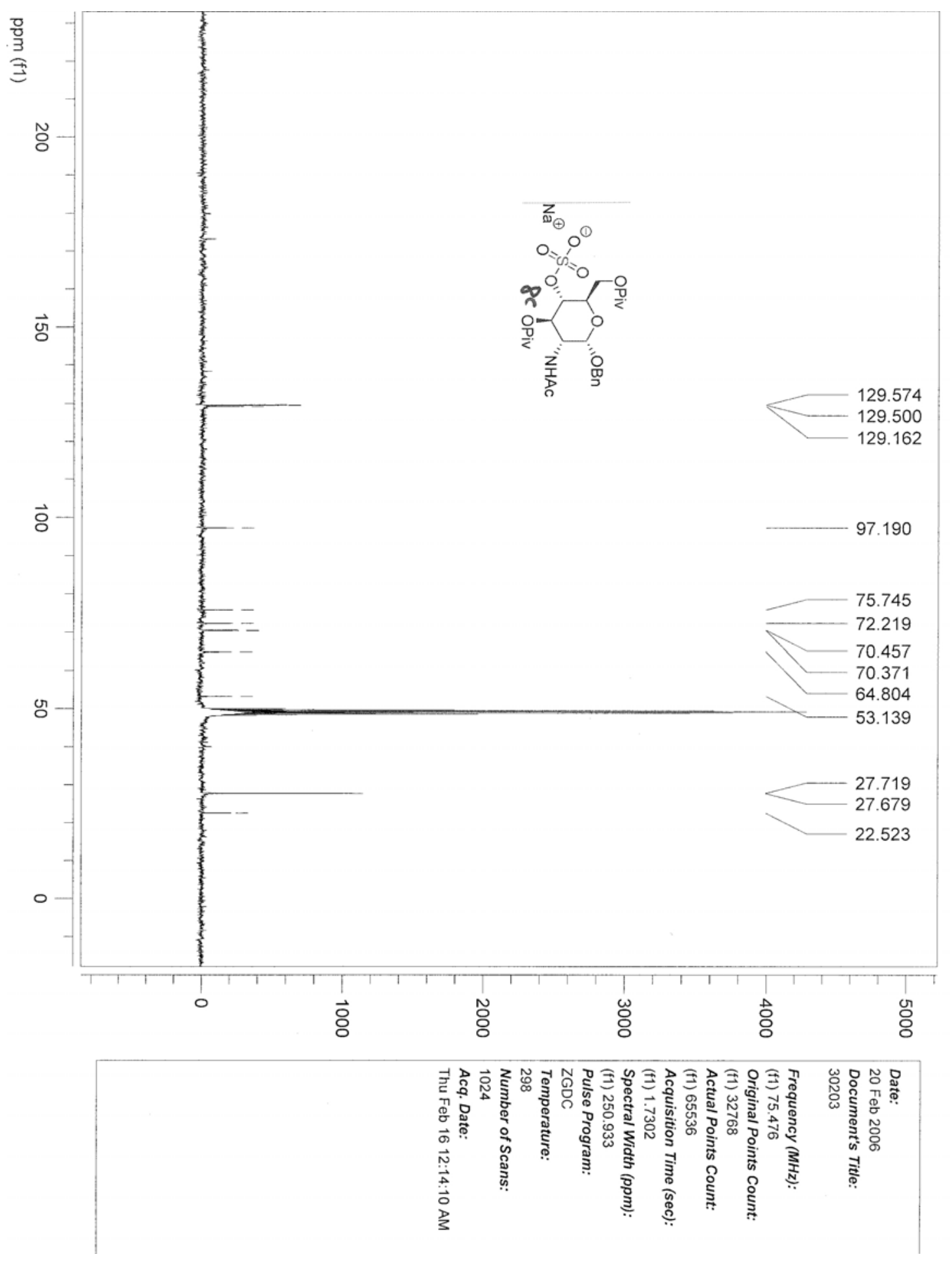


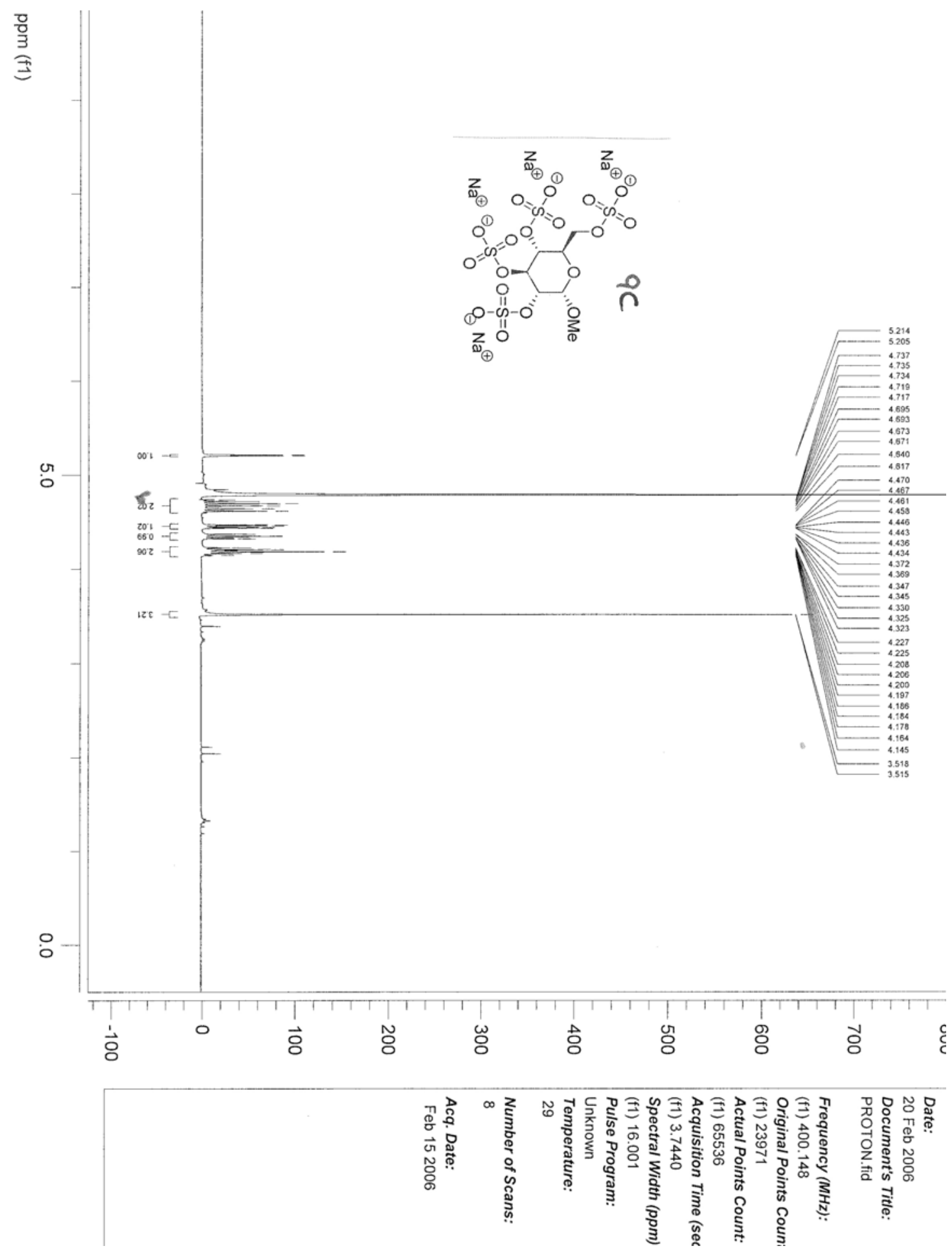




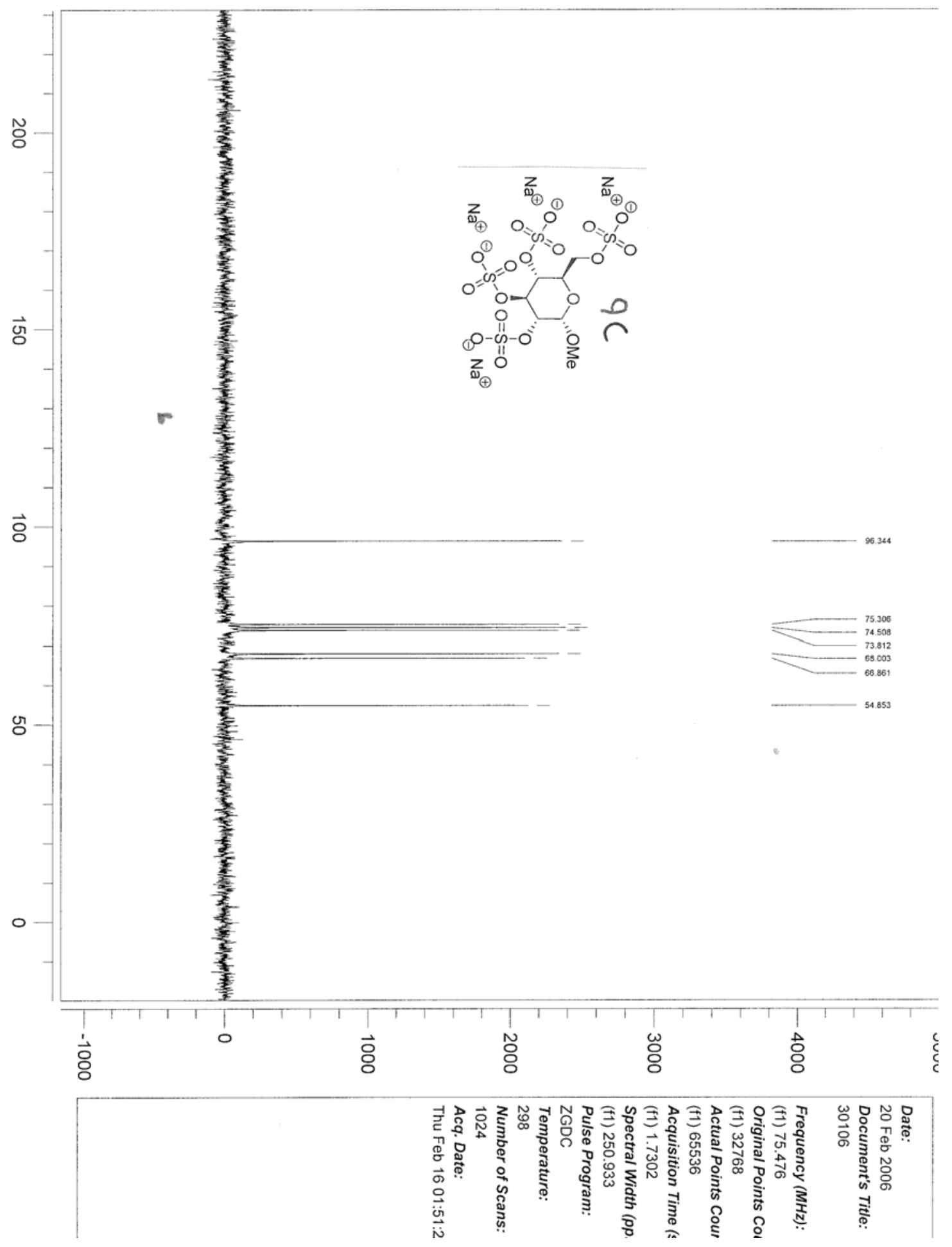




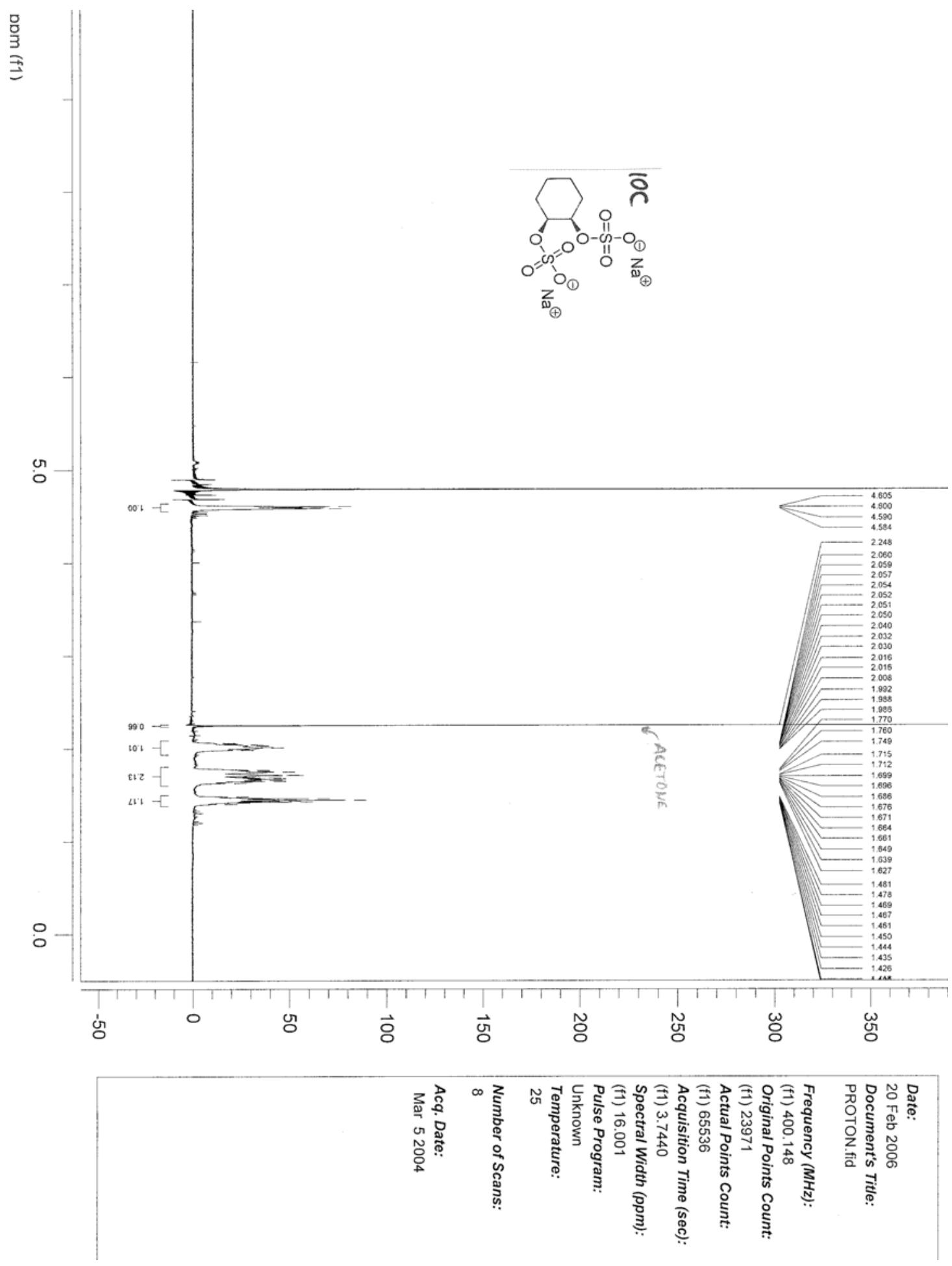



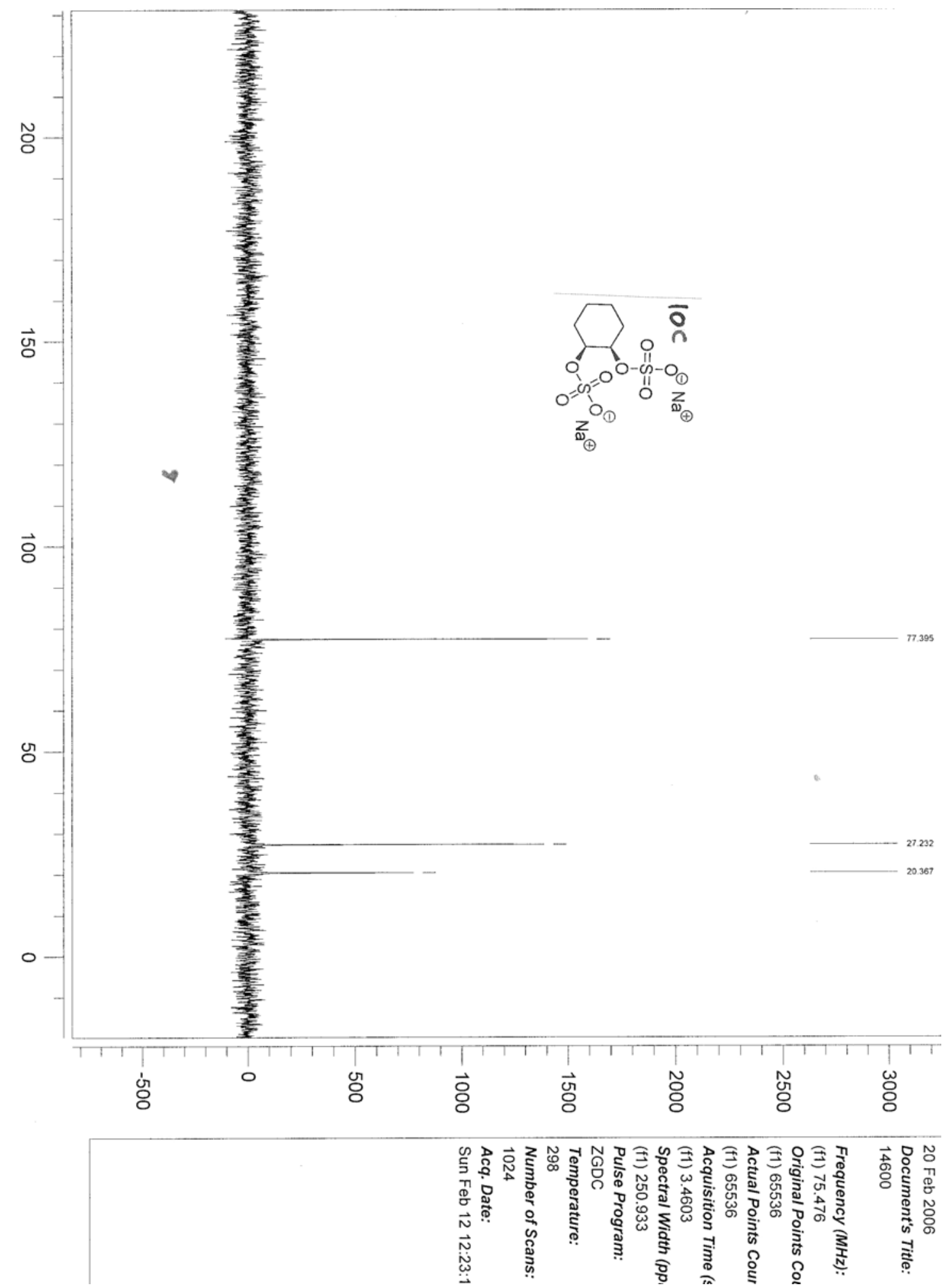


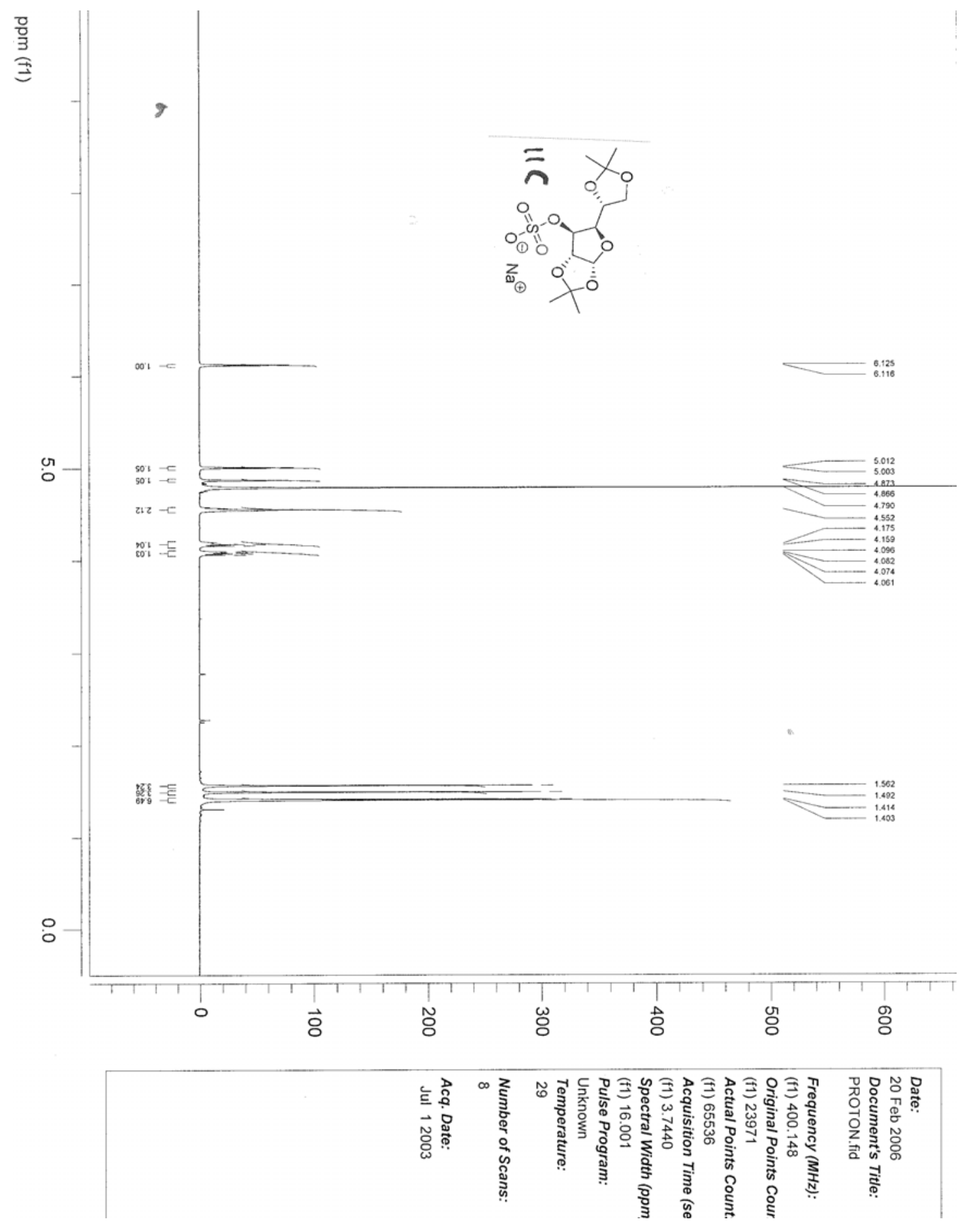




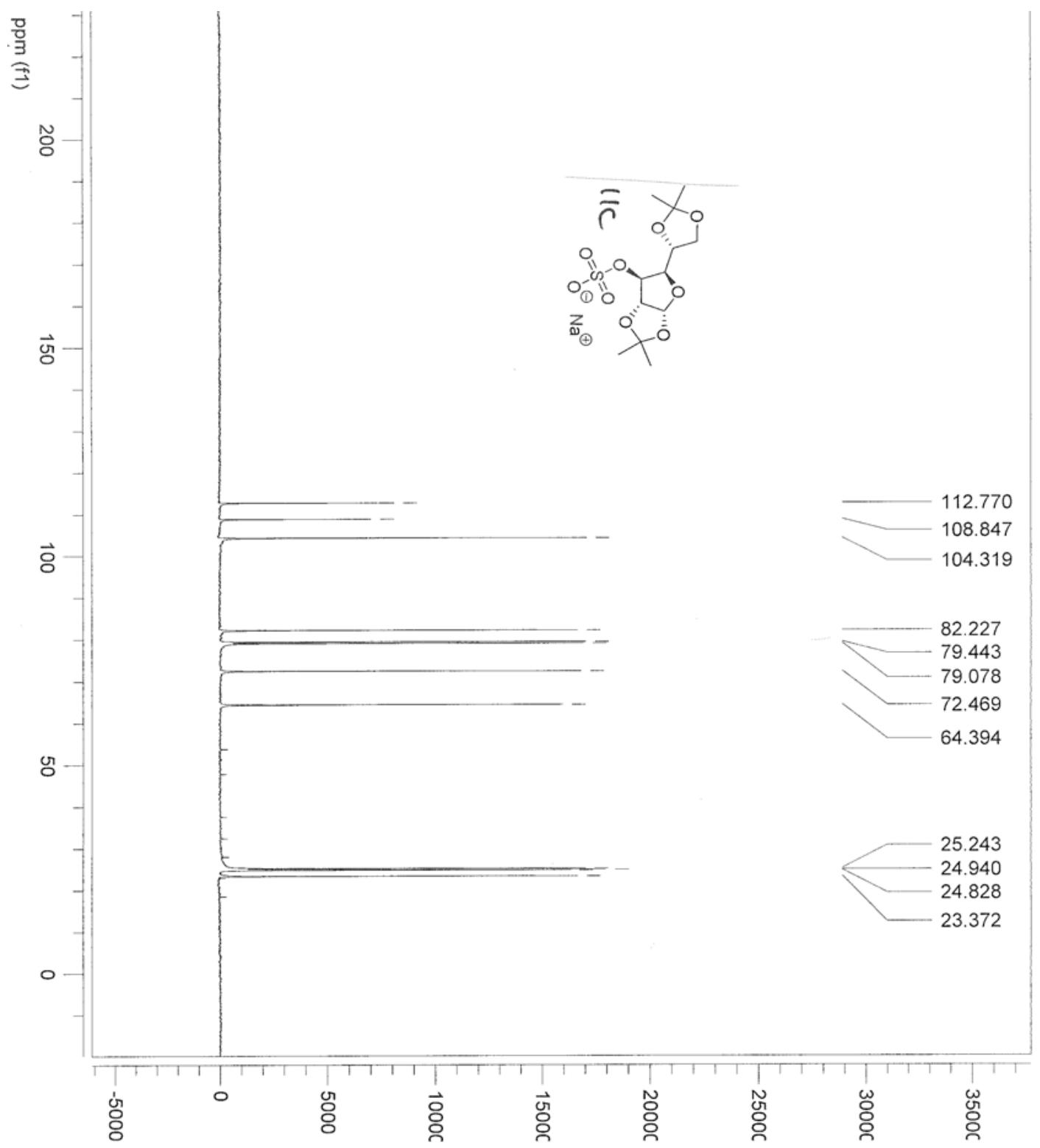

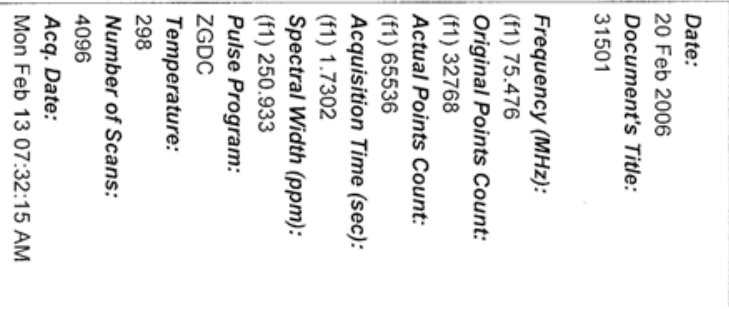




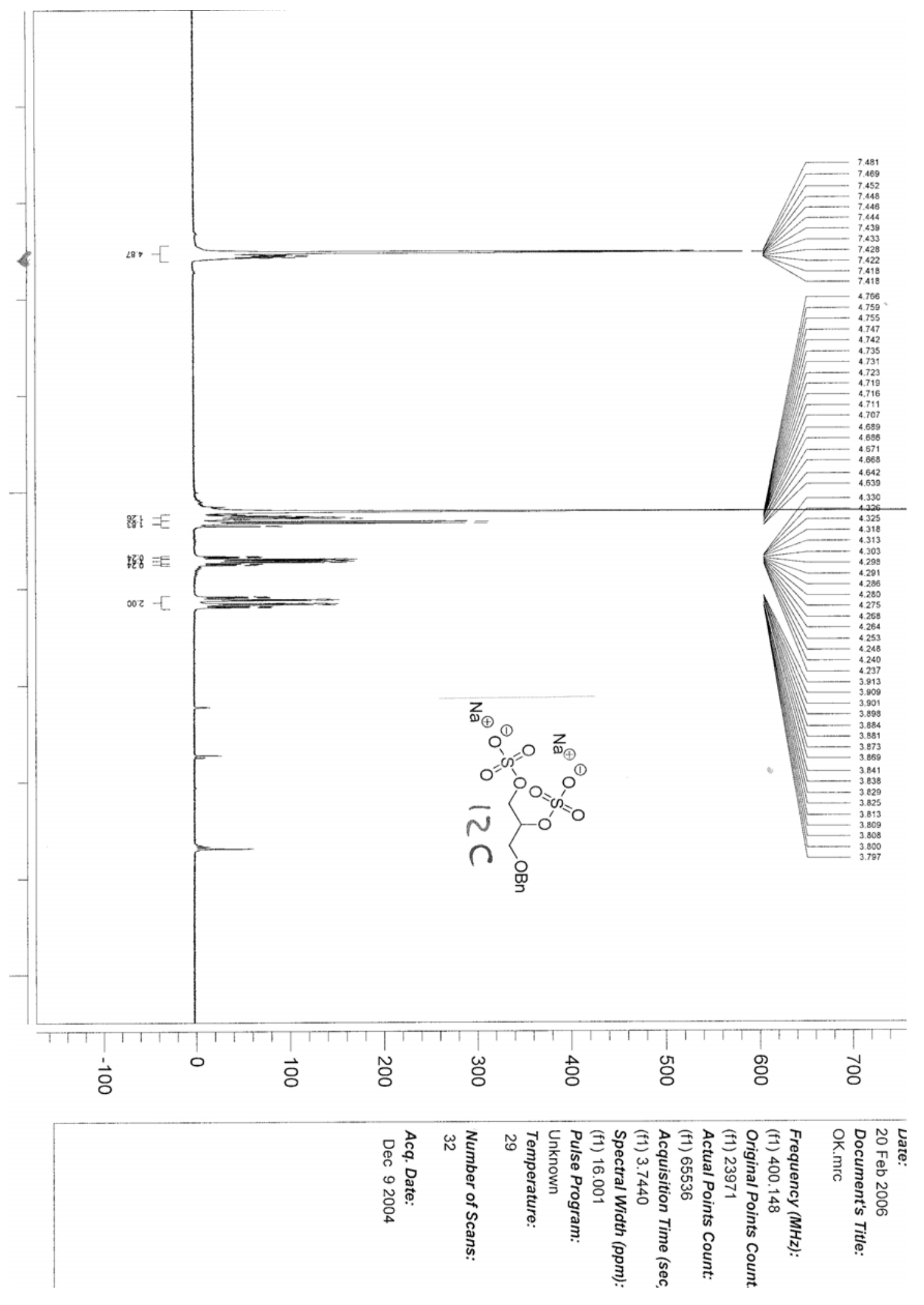




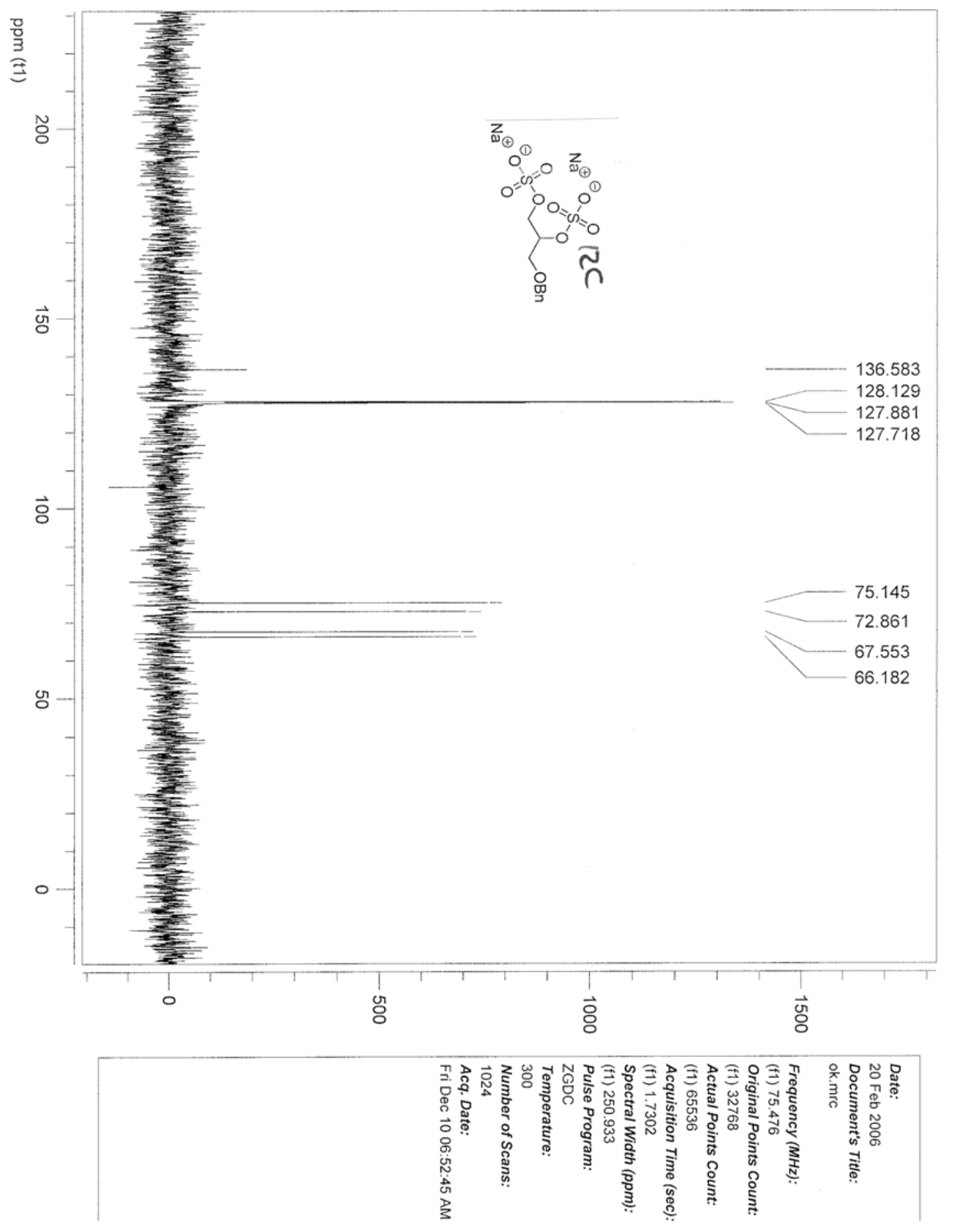




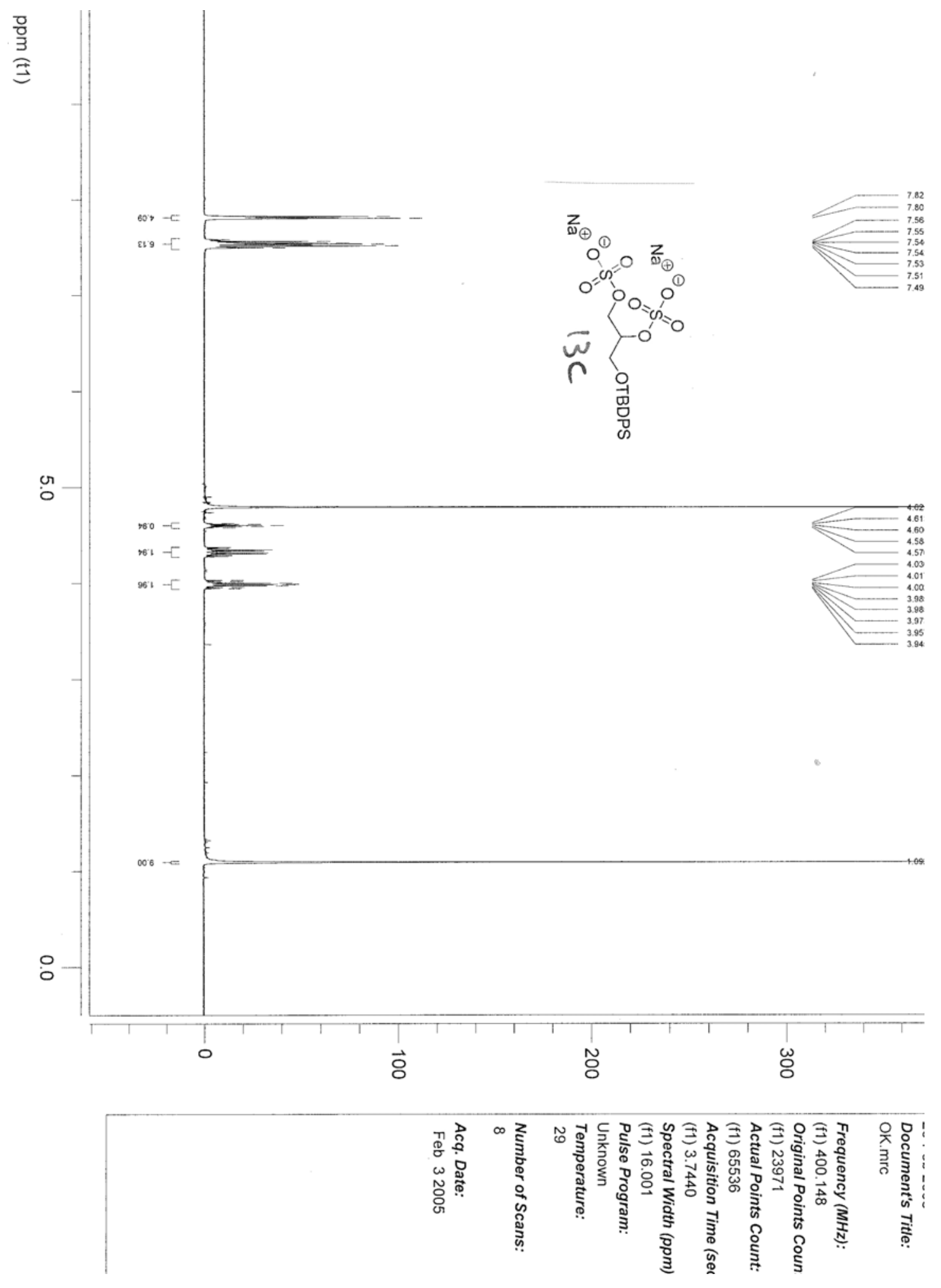




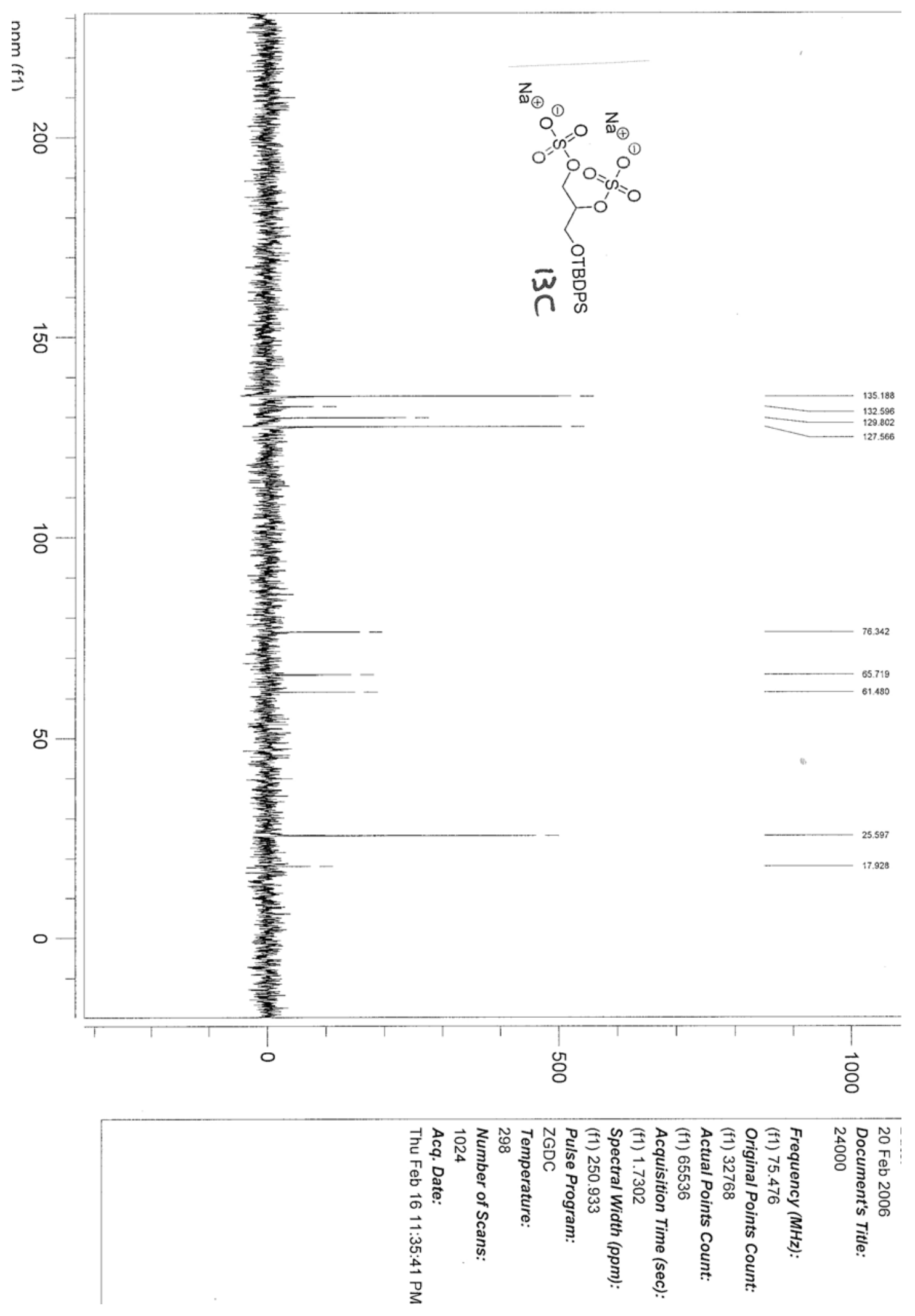




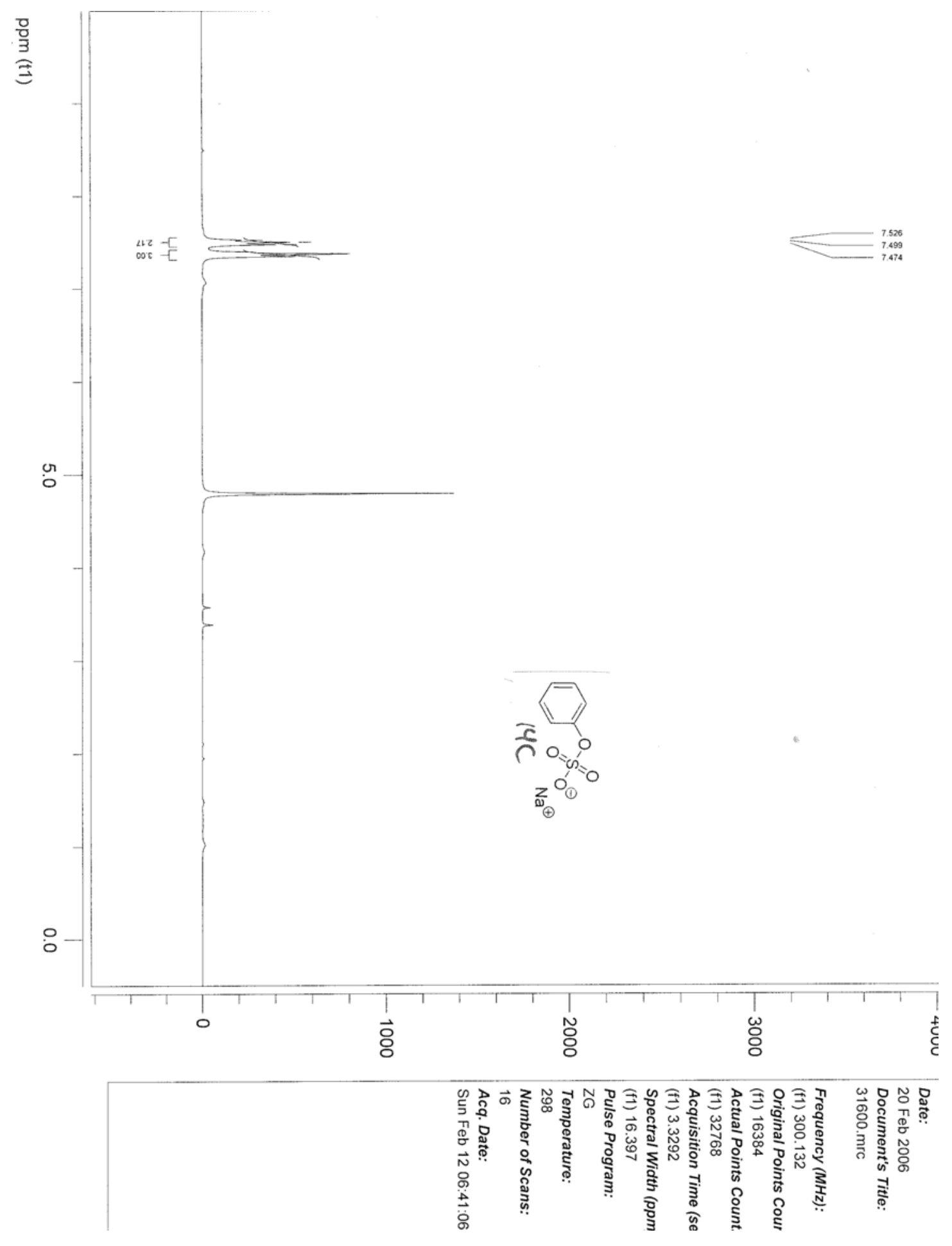




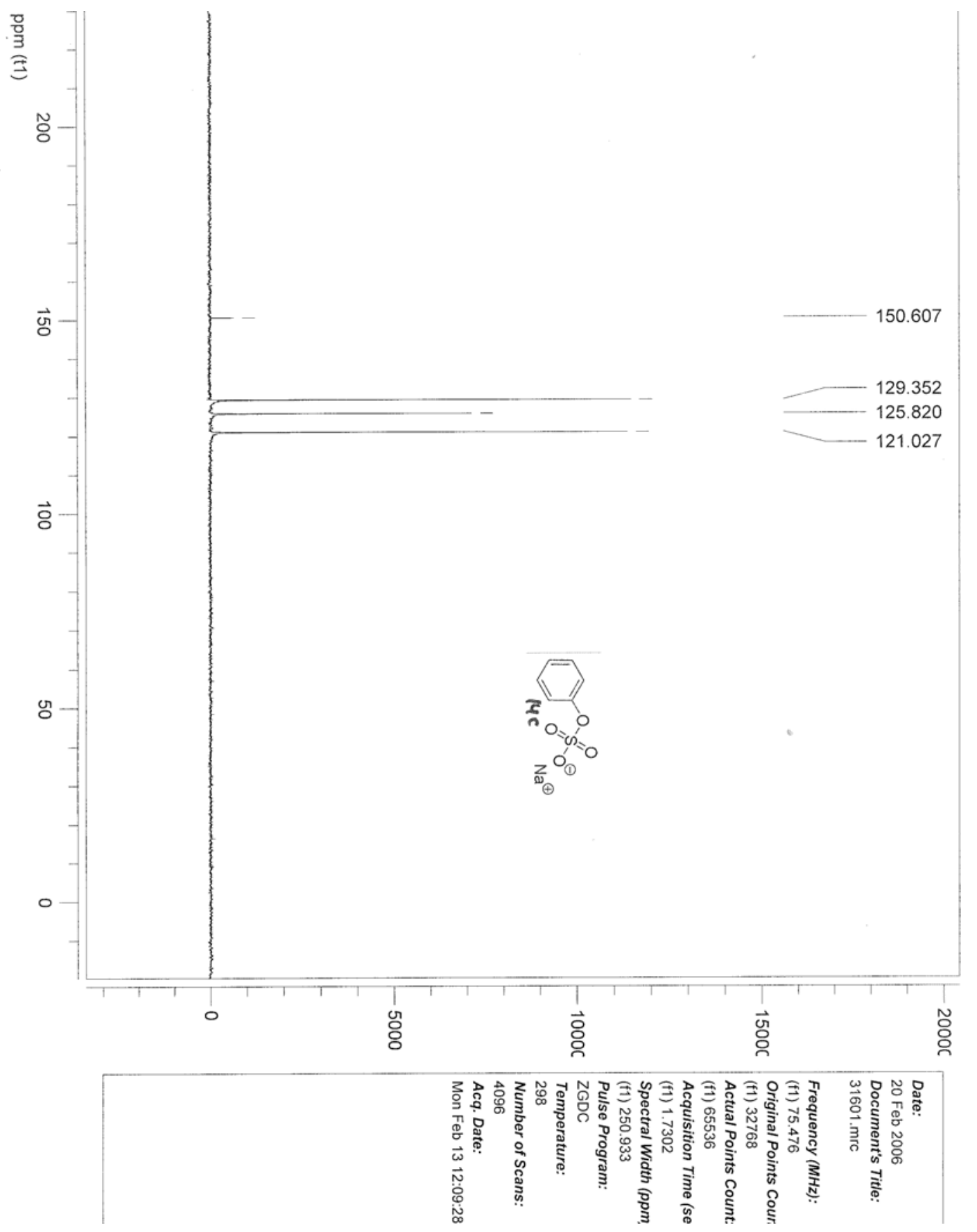




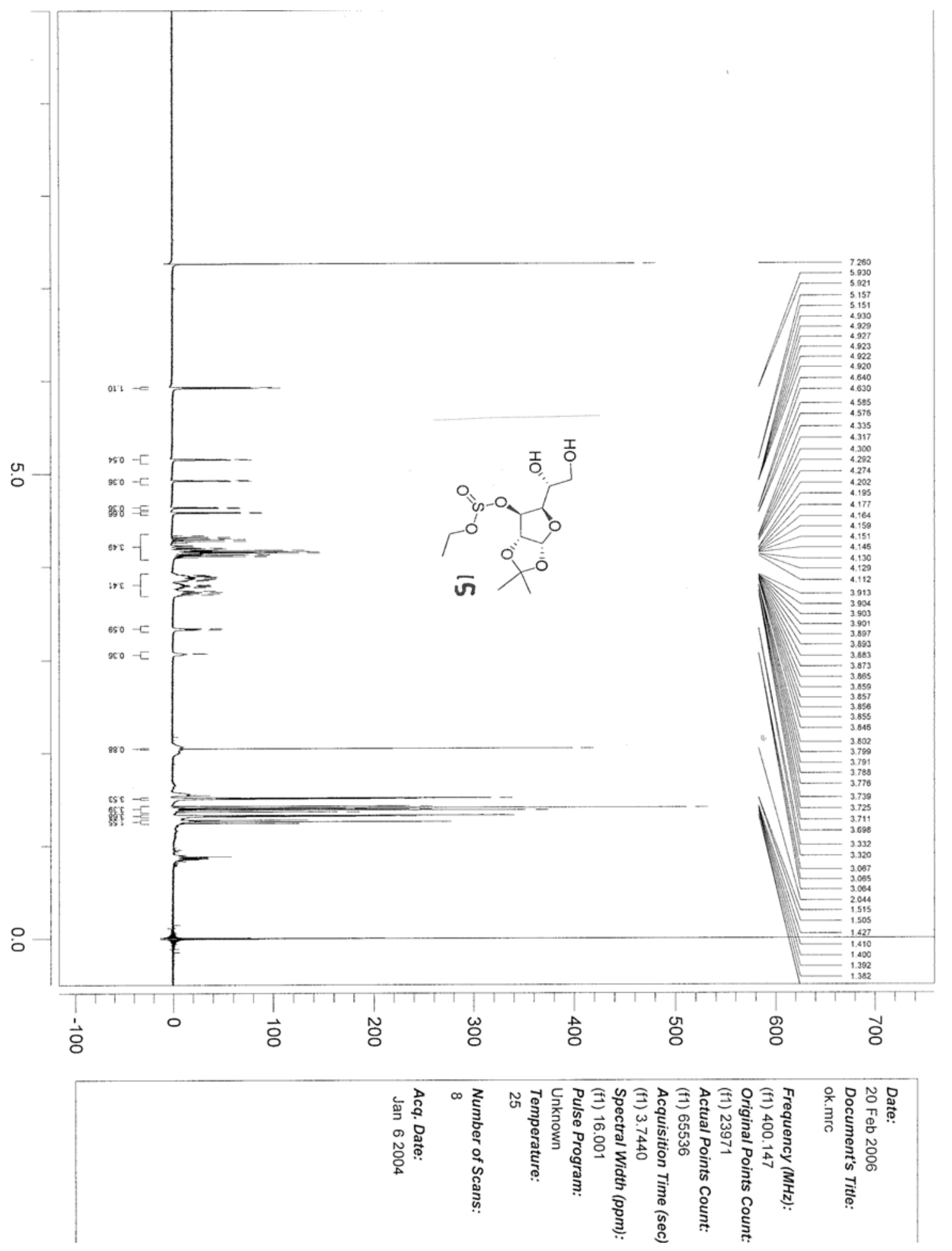




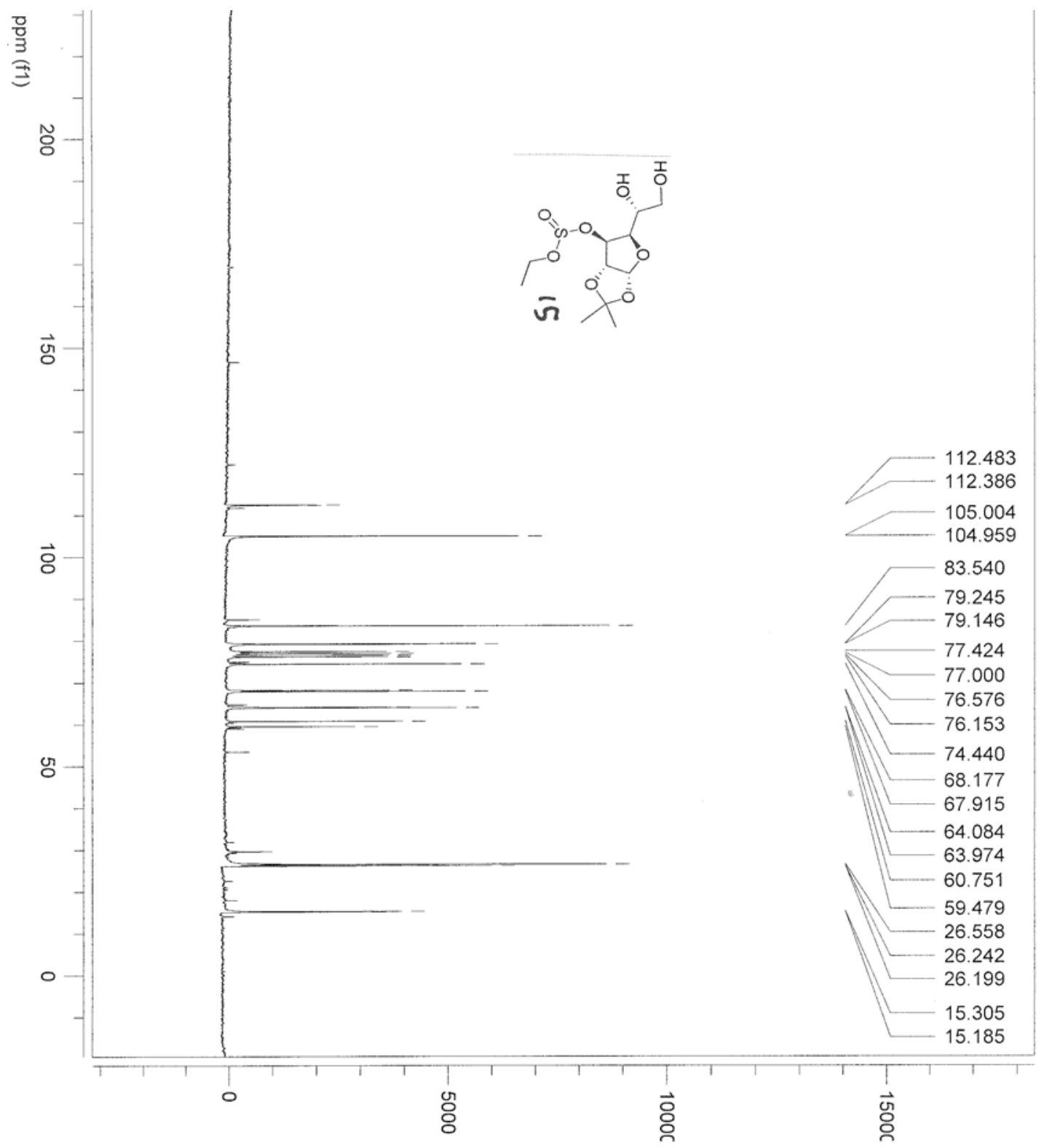

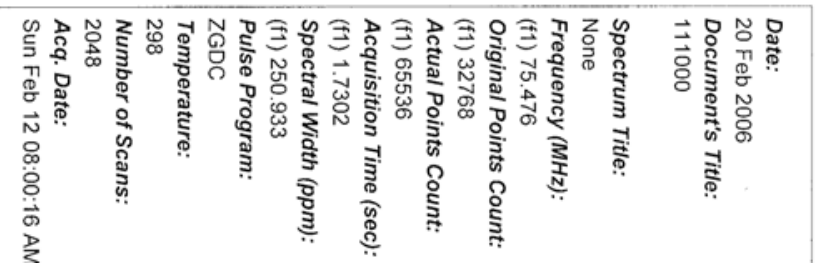



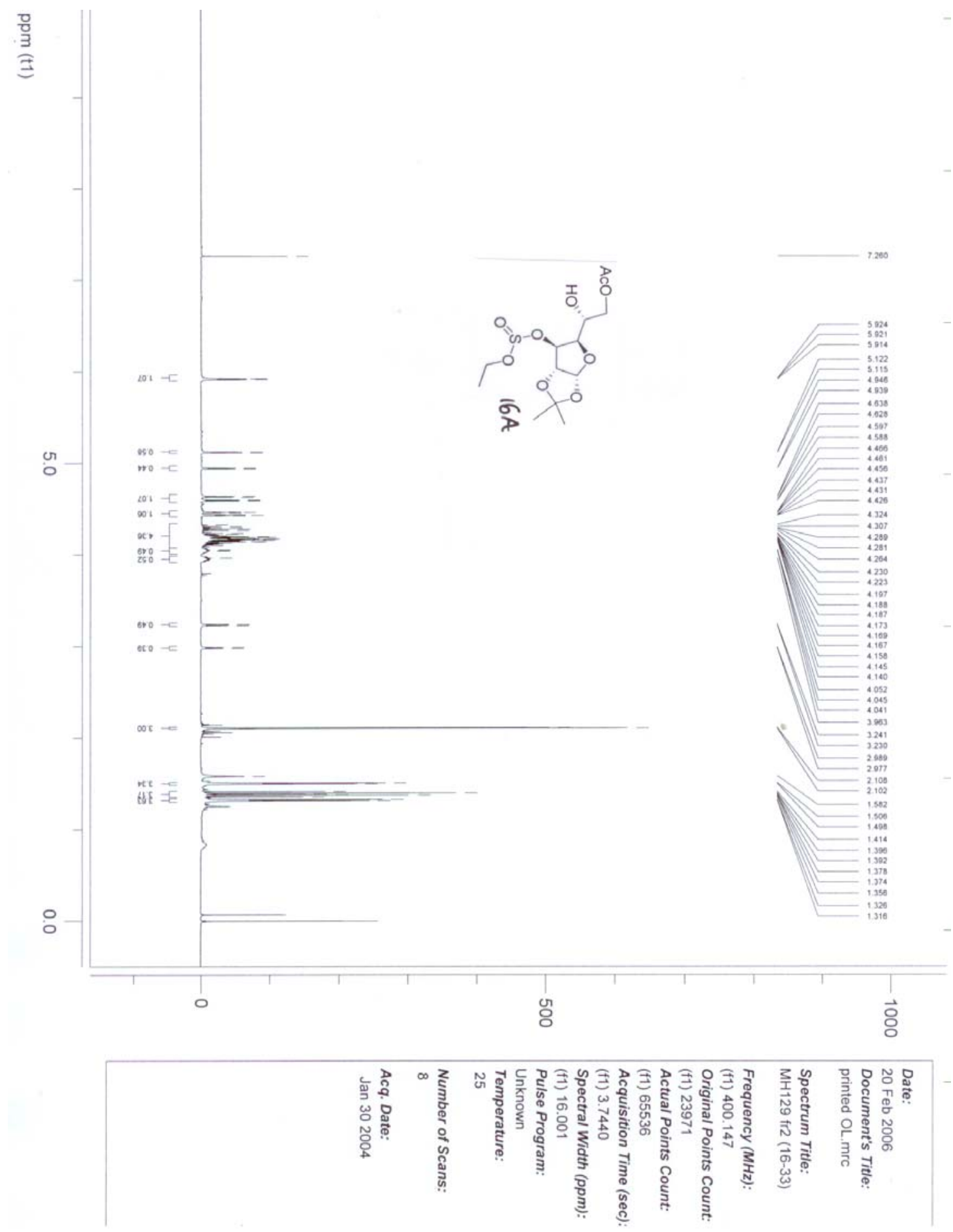


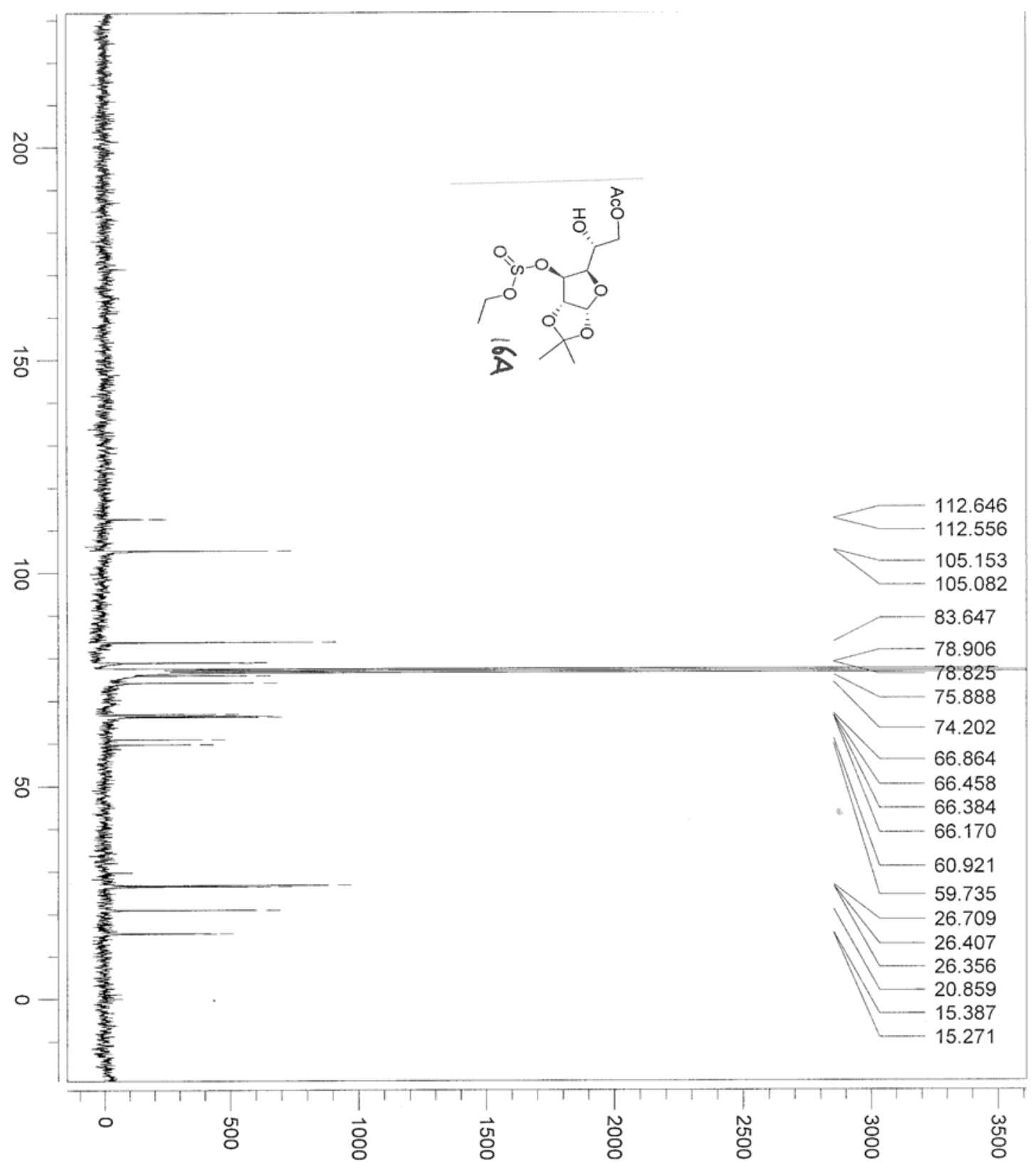

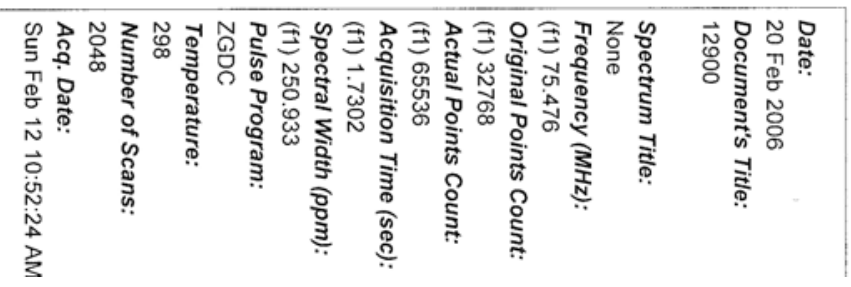

